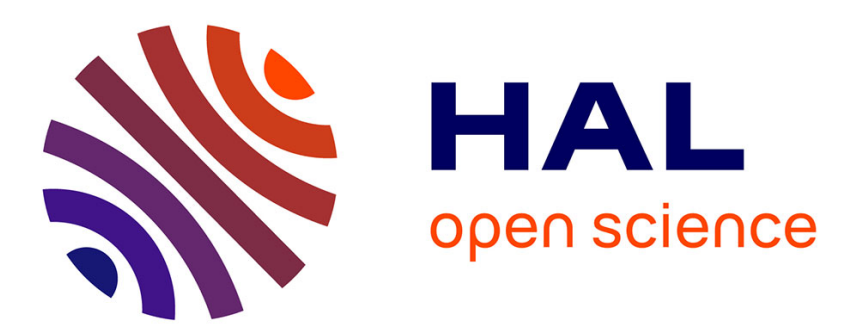

\title{
Space of 2D elastic materials: a geometric journey
}

Boris Desmorat, Nicolas Auffray

\section{To cite this version:}

Boris Desmorat, Nicolas Auffray. Space of 2D elastic materials: a geometric journey. 2018. hal01906468

\section{HAL Id: hal-01906468 \\ https://hal.science/hal-01906468}

Preprint submitted on 26 Oct 2018

HAL is a multi-disciplinary open access archive for the deposit and dissemination of scientific research documents, whether they are published or not. The documents may come from teaching and research institutions in France or abroad, or from public or private research centers.
L'archive ouverte pluridisciplinaire HAL, est destinée au dépôt et à la diffusion de documents scientifiques de niveau recherche, publiés ou non, émanant des établissements d'enseignement et de recherche français ou étrangers, des laboratoires publics ou privés. 


\title{
Space of 2D elastic materials: a geometric journey
}

\section{B. Desmorat • N. Auffray}

Received: date / Accepted: date

\begin{abstract}
In this paper, we describe geometrically the elastic material domain in terms of invariants of the integrity basis (including the positive definiteness condition), prove a theoretical link between those polynomial invariants and the Kelvin invariants of the elasticity tensor, to finally introduce the concept of design transformation which leads to elastic materials subdomains with identical Kelvin invariants.
\end{abstract}

Keywords 2D elasticity · Invariants · Integrity basis · Eigenvalues

\section{Contents}

1 Introduction . . . . . . . . . . . . . . . . . . . . . . . . . . . 2

2 Space of linear elastic materials . . . . . . . . . . . . . . . . . . . . . . 4

3 Decomposition of second-order symmetric tensors space . . . . . . . . . . . . . 6

4 Invariant description of $\mathbb{E}$ la . . . . . . . . . . . . . . . . . . . . . 8

5 Invariant based positive definiteness condition for elastic materials . . . . . . . . . . 12

6 An explicit link between Boehler and Kelvin invariants . . . . . . . . . . . . . . . . . 16

7 Design transformations . . . . . . . . . . . . . . . . . . . . . . 18

A Normal forms ... . . . . . . . . . . . . . . . . . . . . . . . . . 22

B Quaternionic parametrization of rotations . . . . . . . . . . . . . . . . . 22

C Proofs of propositions 7.4 and 7.5 . . . . . . . . . . . . . . . . . . . 25

D On reduced anisotropic invariants . . . . . . . . . . . . . . . . . . 26

B. Desmorat

Sorbonne Université, CNRS, Institut Jean Le Rond d'Alembert, UMR 7190, 75005 Paris, France

E-mail: boris.desmorat@sorbonne-universite.fr

N. Auffray

MSME, Université Paris-Est, Laboratoire Modélisation et Simulation Multi Echelle,MSME UMR 8208 CNRS, 5 bd Descartes, 77454 Marne-la-Vallée, France

E-mail: Nicolas.Auffray@u-pem.fr 


\section{Introduction}

In linear elasticity, it is customary to describe an elastic material by its tensor specified with respect to some basis. Such a way to describe elastic materials is not satisfactory since rotating the matter produces, with respect to the same basis, another elasticity tensor describing the same elastic material. Hence an elastic material is not defined by an unique tensor but rather by the collection of all elastic tensors related by orthogonal transformations. This idea can be condensed by describing elastic materials by a collection of quantities which are invariant with respect to orthogonal transformations. These quantities are often simply reffered to as the invariants of the elasticity tensor [9,31,5,22]. A basis of invariants that are polynomial in terms of tensor components is called an Integrity Basis $(\mathcal{I B})$. Elements of $\mathcal{I B}$, also reffered to as Boehler invariants [9], satisfy polynomials relations defining the set of elastic materials as a domain within a higher dimensional space. Points of this domain uniquely defined elastic materials, and their symmetry classes is encoded in the the topology of the domain. In the case of planar elasticity the domain of elastic materials lives in $\mathbb{R}^{5}$ (the situation is much more complicated for 3D elasticity and will not be discussed here).

On the other hand, the Kelvin representation, originaly due to Kelvin [27] and appearing again in the litterature of the 80's [25,20], allows for the definition of 3 polynomial invariants (the elementary symmetric functions), denoted Kelvin invariants, which are uniquely related to the 3 eigenvalues of such tensorial representation. Such invariants are used in mechanics with different purposes : dissymmetric elastic behaviour in tension and in compression [10], yield and strength criteria $[1,14]$, continuum damage mechanics $[6,15,12,13,16,19]$... Moreover, the link between the eigenvalues of the Kelvin decomposition of $2 \mathrm{D}$ elasticity tensor and the polar formalism [30,29] was described in [11].

In this paper, devoted to the $2 \mathrm{D}$ case, we will describe geometrically the elastic material domain in terms of invariants of the integrity basis (including the positive definiteness condition), prove a theoretical link between the two non-equivalent sets of polynomial Kelvin and Boehler invariants, to finally introduce the concept of design transformation which leads to elastic materials subdomains with identical Kelvin invariants.

\section{Organization of the paper}

First, section 2 is devoted to a brief description of the space of $2 \mathrm{D}$ linear elastic material. Next, in section 3, the decomposition of second-order symmetric tensor space into deviatoric and spheric subspaces is introduced. In section 4 , the Clebsh-Gordan harmonic decomposition of elasticity tensors is detaiiled and the integrity basis defined accordingly. The section 5 the definite positiveness conditions expressed in terms of the invariants of the integrity basis are provided, and geometrical description of the elastic material domain is proposed. In section 6 , an explicit link between Boehler and Kelvin invariants, that makes use of the spheric direction introduced in section 3 is shown. And, finally, we introduce in section 7 the concept of design transformation which transforms elastic materials while preserving the eigenvalues of the Kelvin representation of an elasticity tensor. An explicitly characterization of geometric domains obtained by all possible design 
transformations for an initially isotropic material is studied, with an application to the specific case of pentamode materials.

\section{Notations}

Throughout this paper, the physical space is modelled on the Euclidean space $\mathcal{E}^{2}$ with $\mathrm{E}^{2}$ its associated vector space. Once an arbitrary reference point chosen those spaces can be associated and $\mathcal{P}=\left\{\underline{\mathrm{e}}_{1}, \underline{\mathrm{e}}_{2}\right\}$ will denote an orthonormal basis of $\mathrm{E}^{2}$. For forthcoming need, let also defined $\mathcal{K}=\left\{\underline{\hat{e}}_{1}, \underline{\hat{e}}_{2}, \underline{\hat{e}}_{3}\right\}$ the orthonormal canonical basis of $\mathbb{R}^{3}, \mathcal{K}$ will be referred to as the Kelvin basis.

\section{Tensor spaces:}

- $\mathbb{T}$ denote a tensor space;

$-S^{2}\left(\mathbb{R}^{n}\right)$ is the space of symmetric second-order tensors in $n$-D.

- $\mathbb{K}^{n}$ is the space of $n$-th order completely symmetric and traceless tensors on $\mathbb{R}^{2}$, called harmonic tensors.

Tensors of order ranking from 0 to 4 are denoted, respectively, by $\alpha, \underset{\sim}{\mathrm{v}}, \underset{\sim}{\mathrm{a}}, \underset{\approx}{\mathrm{A}}$. The simple, double and fourth-order contractions are written $\cdot,:$, : respectively. In components, with respect to $\mathcal{P}$, these notations correspond to

$\underline{\mathrm{u}} \cdot \underline{\mathrm{v}}=u_{i} v_{i}, \quad \underset{\sim}{\mathrm{a}}: \underset{\sim}{\mathrm{b}}=a_{i j} b_{i j}, \quad(\underset{\approx}{\mathrm{A}}: \underset{\approx}{\mathrm{B}})_{i j k l}=A_{i j p q} B_{p q k l}, \quad(\underset{\approx}{\mathrm{A}}:: \underset{\approx}{\mathrm{B}})=A_{p q r s} B_{p q r s}$

where the Einstein summation on repeated indices is understood. When needed, index symmetries of both spaces and their elements are expressed as follows: (..) indicates invariance under permutations of the indices in parentheses, ..... indicate symmetry with respect to permutations of the underlined blocks.

Tensor products:

- $\otimes$ stands for the classical product, and $\otimes^{n}$ indicates its $n$-th power;

$-S^{2}$ denotes its the completely symmetrized product, and $S^{n}$ its extension to product of $n$ elements;

- $\bar{Q}$ indicates the twisted tensor product defined by:

$$
(\underset{\sim}{\mathrm{a}} \underset{\sim}{\mathrm{b}})_{i j k l}=\frac{1}{2}\left(a_{i k} b_{j l}+a_{i l} b_{j k}\right)
$$

Special tensors:

- 1 the second-order identity tensor;

$-\underset{\sim}{\mathrm{I}}=\underset{\sim}{1} \underline{\bar{\otimes}} 1$ the fourth-order identity tensor of $S^{2}\left(\mathbb{R}^{n}\right)$;

$-\underset{\widetilde{K}}{\mathrm{~K}}=\frac{1}{2} \underset{\sim}{1} \otimes \underset{\sim}{1}$ the spheric projector;

$-\underset{\approx}{\underset{\mathrm{J}}{\mathrm{I}}}=\underset{\approx}{\mathrm{I}}-\underset{\approx}{\mathrm{K}}$ the deviatoric projector. 
Matrix spaces:

$-\mathcal{M}_{n}$ is the space of $n \times n$ dimensional square matrices ;

- $\mathcal{M}_{n}^{S}$ is the space of $n \times n$ dimensional symmetric square matrices ;

$-\mathcal{M}_{p, q}$ is the space of $p \times q$ rectangular matrices.

Groups:

The following matrix groups are considered in the paper:

- GL $(d)$ : the group of all linear invertible transformations of $\mathbb{R}^{d}$, i.e. $\mathrm{F} \in \mathrm{GL}(d)$ iff $\operatorname{det}(\mathrm{F}) \neq 0$

- $\mathrm{O}(d)$ : the orthogonal group, that is the group of all isometries of $\mathbb{R}^{d}$ i.e. $\mathrm{Q} \in$ $\mathrm{O}(d)$ iff $\mathrm{Q} \in \mathrm{GL}(d)$ and $\mathrm{Q}^{-1}=\mathrm{Q}^{T}$, where the superscript ${ }^{T}$ denotes the transposition.

- $\mathrm{SO}(d)$ : the special orthogonal group, i.e. the subgroup of $\mathrm{O}(d)$ consisting of transformations satisfying $\operatorname{det}(\mathrm{Q})=1$.

Let detail the case $d=2$. As a matrix group, $\mathrm{O}(2)$ can be generated by:

$$
\mathrm{R}(\theta)=\left(\begin{array}{cc}
\cos \theta & -\sin \theta \\
\sin \theta & \cos \theta
\end{array}\right), 0 \leq \theta<2 \pi, \quad \text { and } \quad \mathrm{P}\left(\underline{\mathrm{e}}_{2}\right)=\left(\begin{array}{cc}
1 & 0 \\
0 & -1
\end{array}\right)
$$

in which $\mathrm{R}(\theta)$ is a rotation by angle $\theta$ and $\mathrm{P}\left(\underline{\mathrm{e}}_{2}\right)$ is the reflection across the $x$ axis. $\mathrm{SO}(2)$ corresponds to the group of plane rotations generated by $\mathrm{R}(\theta)$. The following finite subgroups of $\mathrm{O}(2)$ will be used :

- Id, the identity group;

- $\mathrm{Z}_{k}$, the cyclic group with $k$ elements generated by $\mathrm{R}(2 \pi / k)$;

$-\mathrm{D}_{k}$, the dihedral group with $2 k$ elements generated by $\mathrm{R}(2 \pi / k)$ and $\mathrm{P}_{\underline{\mathrm{e}}_{2}}$.

Miscellaneous notations:

- IB means Integrity Basis.

$-\simeq$ denotes hereafter an isomorphism.

$-\mathcal{S}^{n}$ denotes he unit $n$-sphere defined as:

$$
\mathcal{S}^{n}=\left\{x \in \mathbb{R}^{n+1}:\|x\|=r\right\} .
$$

$-\mathcal{L}(E, F)$ indicates the space of linear applications from $E$ to $F$;

$-\mathcal{L}(E)$ indicates the space of linear applications from $E$ to $E$;

$-\mathcal{L}^{\mathcal{S}}(E)$ indicates the space of self-adjoint linear applications on $E$;

\section{Space of linear elastic materials}

2.1 The space elasticity tensors

In the field of linear elasticity, the constitutive law is a local linear relation between the second-order symmetric Cauchy stress tensor $\underset{\sim}{\sigma}$ and the second-order symmetric infinitesimal strain tensor $\underset{\sim}{\varepsilon}$ :

$$
\sigma_{i j}=C_{i j k l} \varepsilon_{k l}
$$


$\sigma$ and $\underset{\sim}{\varepsilon}$ belong to $S^{2}\left(\mathbb{R}^{2}\right)$, the space of bi-dimensional symmetric second-order tensors. As a consequence the elasticity tensor possesses minor index symmetries:

$$
C_{i j k l}=C_{j i k l}=C_{i j l k}
$$

which are condensed in the notation: $C_{(i j)(k l)}$. Due to the potential energy associated to the elastic behavior another index symmetry has to be taken into account:

$$
C_{i j k l}=C_{k l i j}
$$

This so-called major symmetry is encoded in the notation: $C_{i j} \underline{k l}$. Hence, combined with the minor ones, we obtain the elastic index symmetries: $\underline{C_{(i j)}} \underline{(k l)}$. The vector space of $2 \mathrm{D}$ elasticity tensors is defined $\mathrm{as}^{1}$ :

$$
\mathbb{E} \text { la }:=\left\{\underset{\approx}{\mathrm{C}} \in \otimes^{4} \mathbb{R}^{2} \mid C_{\underline{(i j)}} \underline{\underline{(k l)}}\right\}, \quad \operatorname{dim} \mathbb{E} \text { la }=6
$$

and can also be viewed as

$$
\mathbb{E l a}=\mathcal{L}^{s}\left(S^{2}\left(\mathbb{R}^{2}\right)\right)
$$

For being admissible, an elasticity tensor, considered as a quadratic form on $S^{2}\left(\mathbb{R}^{2}\right)$, should further be positive definite, meaning that its eigenvalues $\lambda_{i}$ should verify

$$
\exists M \in \mathbb{R}^{*+}, \quad 0<\lambda_{i} \leq M
$$

2.2 From active physical rotations to elastic materials

Consider $\mathrm{O}(2)$ the set of $2 \mathrm{D}$ isometric transformations. Its action on an element $\underset{\approx}{\mathrm{T}}$ of $\mathbb{E}$ la gives a new element $\underset{\approx}{\overline{\mathrm{T}}}$ of $\mathbb{E}$ la,

$$
\underset{\approx}{\overline{\mathrm{T}}}=\mathrm{Q} \star \underset{\approx}{\mathrm{T}}
$$

in which the star product $\star$ stands for the standard tensorial action. In components, with respect to $\mathcal{P}$, this action can be detailed:

$$
\bar{T}_{i j k l}=Q_{i p} Q_{j q} Q_{k r} Q_{l s} T_{p q r s}
$$

The nature of a material does not change when it is subjected to a rotation or a flip (mirror isometry though a line). On the contrary the elasticity tensor will change with respect to a given reference frame, for instance $\mathcal{P}$, so that multiple tensors can be associated to one elastic material.

From a physical point of view, the necessary and sufficient conditions for two elastic tensors $\underset{\approx}{\mathrm{T}_{1}}, \underset{\approx}{\mathrm{T}_{2}} \in \mathbb{E}$ la to represent the same elastic material, denoted $\underset{\approx}{\mathrm{T}_{1}} \sim$ $\mathrm{T}_{2}$, writes

$$
\underset{\approx}{\mathrm{T}_{1}} \sim \underset{\approx}{\mathrm{T}} \quad \Leftrightarrow \quad \exists \mathrm{Q} \in \mathrm{O}(2) \mid \underset{\approx}{\mathrm{T}}=\mathrm{Q} \star \underset{\approx}{\mathrm{T}}
$$

1 Even if obvious, it is worth mentioning that both the stiffness tensor and its inverse, the compliance tensor, belong to the same vector space. Hence in the following no physical interpretation (stiffness or compliance) will be given to elements of $\mathbb{E}$ la. 
The collection of all elasticity tensors that describe the same elastic material is a geometric object called the orbit of $\mathrm{T}_{1}$ and defined by

$$
\mathcal{O}\left(\underset{\approx}{\mathrm{T}_{1}}\right)=\left\{\underset{\approx}{\mathrm{T}} \in \mathbb{E l a}, \exists \mathrm{Q} \in \mathrm{O}(2) \mid \underset{\approx}{\mathrm{T}}=\mathrm{Q} \star \underset{\approx}{\mathrm{T}_{1}}\right\}
$$

For some transformation, the resulting tensor is identical to the original one. The set of orthogonal transformations letting $\underset{\approx}{\mathrm{T}_{1}}$ invariant constitutes its symmetry group:

$$
\mathrm{G}_{\mathrm{T}_{1}}:=\left\{\mathrm{Q} \in \mathrm{O}(2), \mid \underset{\approx}{\mid \mathrm{T}_{1}}=\mathrm{Q} \star \underset{\approx}{\mathrm{T}_{1}}\right\}
$$

Let consider the following equivalence relation among elements of $\mathbb{E}$ la,

$$
\underset{\approx}{\mathrm{T}_{1}} \approx \underset{\approx}{\mathrm{T}_{2}} \Leftrightarrow \quad \exists \mathrm{Q} \in \mathrm{O}(2) \mid \underset{\approx}{\mathrm{G}_{\mathrm{T}_{1}}}=\mathrm{QG}_{\underset{\mathrm{T}_{2}}{ }} \mathrm{Q}^{-1}
$$

Through this relation two tensors are equivalent if their symmetry group are conjugate. The equivalence classes for this relation are called strata. More specifically, in what follows $\Sigma_{[G]}$ will denote the equivalence class of elasticity tensors having their symmetry group conjugate to $G$. In other words, $[G]$ is the symmetry class of the elements of the stratum $\Sigma_{[G]}[4,3]$. The space of $2 \mathrm{D}$ elasticity tensors is divided into 4 strata $[18,31,3]$ :

$$
\mathbb{E l a}=\Sigma_{\left[\mathrm{Z}_{2}\right]} \cup \Sigma_{\left[\mathrm{D}_{2}\right]} \cup \Sigma_{\left[\mathrm{D}_{4}\right]} \cup \Sigma_{[\mathrm{O}(2)]}
$$

In mechanical terms, $\Sigma_{\left[\mathrm{Z}_{2}\right]}$ corresponds to the set of biclinic materials, $\Sigma_{\left[\mathrm{D}_{2}\right]}$ to the set of orthotropic materials, $\Sigma_{\left[\mathrm{D}_{4}\right]}$ to the set of tetragonal materials and $\Sigma_{[\mathrm{O}(2)]}$ to isotropic materials [34].

\section{Decomposition of second-order symmetric tensors space}

Since elasticity tensors are linear symmetric applications on $S^{2}\left(\mathbb{R}^{2}\right)$, a first step to describe $\mathbb{E}$ la is to understand the structure of $S^{2}\left(\mathbb{R}^{2}\right)$. This is the aim of the present section.

\subsection{The harmonic basis}

With respect to $\mathcal{P}=\left(\underline{\mathrm{e}}_{1}, \underline{\mathrm{e}}_{2}\right), \underset{\sim}{\mathrm{t}} \in S^{2}\left(\mathbb{R}^{2}\right)$ can be represented by a matrix:

$$
\stackrel{\mathrm{t}}{\sim}=\left(\begin{array}{ll}
t_{11} & t_{12} \\
t_{12} & t_{22}
\end{array}\right)_{\mathcal{P}}
$$

It is possible to turn this second-order symmetric tensor into a genuine vector of $\mathbb{R}^{3}$ by defining the following linear application $\phi: S^{2}\left(\mathbb{R}^{2}\right) \rightarrow \mathbb{R}^{3}$ :

$$
\left\{\begin{array}{l}
\underline{\hat{\mathrm{e}}}_{1}=\phi\left(\underline{\mathrm{e}}_{1} \otimes \underline{\mathrm{e}}_{1}\right) \\
\underline{\mathrm{e}}_{2}=\phi\left(\underline{\mathrm{e}}_{2} \otimes \underline{\mathrm{e}}_{2}\right) \\
\underline{\mathrm{e}}_{3}=\phi\left(\frac{\sqrt{2}}{2}\left(\underline{\mathrm{e}}_{1} \otimes \underline{\mathrm{e}}_{2}+\underline{\mathrm{e}}_{2} \otimes \underline{\mathrm{e}}_{1}\right)\right)
\end{array}\right.
$$


The cannonical basis $\mathcal{K}=\left\{\underline{\hat{\mathrm{e}}}_{i}\right\}$ of $\mathbb{R}^{3}$ will be referred to as the Kelvin basis. With respect to $\mathcal{K}$, we can define the vector $\underline{\hat{\mathrm{t}}}$ image of $\underset{\sim}{\mathrm{t}}$ by $\phi$

$$
\underline{\hat{\mathrm{t}}}=\left(\begin{array}{c}
t_{11} \\
t_{22} \\
\sqrt{2} t_{12}
\end{array}\right)_{\mathcal{K}}
$$

These two representations are isometric since $\underset{\sim}{\mathrm{t}}: \underset{\sim}{\mathrm{t}}=\underline{\hat{\mathrm{t}}} \cdot \underline{\hat{\mathrm{t}}}$. A third representation is possible. This representation is associated to the decomposition of $S^{2}\left(\mathbb{R}^{2}\right)$ into a deviatoric space $\left(\mathbb{K}^{2}\right)$ and a spheric one $\left(\mathbb{K}^{0}\right)$. In formula:

$$
S^{2}\left(\mathbb{R}^{2}\right) \simeq \mathbb{K}^{2} \oplus \mathbb{K}^{0}
$$

Hence any $\underset{\sim}{\mathrm{t}} \in S^{2}\left(\mathbb{R}^{2}\right)$ can be decomposed accordingly. This decomposition, which is unique, is given by the well-known formula:

$$
\underset{\sim}{\mathrm{t}}=\underset{\sim}{\mathrm{d}}+\alpha \underset{\sim}{1}, \quad \text { with } \quad \alpha=\frac{1}{2} \underset{\sim}{\mathrm{t}}: \underset{\sim}{1}, \quad \underset{\sim}{\mathrm{d}}=\underset{\sim}{\mathrm{t}}-\alpha \underset{\sim}{1}
$$

with $\mathrm{d} \in \mathbb{K}^{2}$ and $\alpha \in \mathbb{K}^{0}$. Associated to this decomposition, we define a new basis $\mathcal{H}=\left\{\underline{\hat{\mathrm{f}}}_{1}, \underline{\hat{\mathrm{f}}}_{2}, \underline{\hat{\mathrm{f}}}_{3}\right\}$, with

$$
\underline{\hat{\mathrm{f}}}_{1}=\frac{\sqrt{2}}{2}\left(\underline{\hat{\mathrm{e}}}_{1}-\underline{\hat{\mathrm{e}}}_{2}\right) \quad \underline{\hat{\mathrm{f}}}_{2}=\underline{\hat{\mathrm{e}}}_{3} \quad \underline{\hat{\mathrm{f}}}_{3}=\frac{\sqrt{2}}{2}\left(\underline{\hat{\mathrm{e}}}_{1}+\underline{\hat{\mathrm{e}}}_{2}\right)
$$

This new basis $\mathcal{H}$ will be referred to as the harmonic basis. The passage matrix $\mathrm{P}$ from $\mathcal{K}$ to $\mathcal{H}$ is given by

$$
P_{i j}=\underline{\hat{\mathrm{e}}}_{i} \cdot \hat{\mathrm{f}}_{j} \quad \mathrm{P}=\left(\begin{array}{ccc}
\frac{\sqrt{2}}{2} & 0 & \frac{\sqrt{2}}{2} \\
-\frac{\sqrt{2}}{2} & 0 & \frac{\sqrt{2}}{2} \\
0 & 1 & 0
\end{array}\right)_{\mathcal{K}}
$$

and can be shown to be an element of $\mathrm{O}(3) \backslash \mathrm{SO}(3)$. With respect to $\mathcal{H}, \hat{\mathrm{t}}$ is expressed as

$$
\underline{\hat{\mathrm{t}}}=\sqrt{2}\left(\begin{array}{c}
\frac{t_{11}-t_{22}}{2} \\
t_{12} \\
\frac{t_{11}+t_{22}}{2}
\end{array}\right)_{\mathcal{H}}
$$

$\frac{t_{11}+t_{22}}{2}$ and $\left(\frac{t_{11}-t_{22}}{2}, t_{12}\right)^{T}$ corresponds respectively to the spheric and deviatoric parts of $\hat{\underline{t}}$.

The relations between these different bases are summed up in the following diagram:

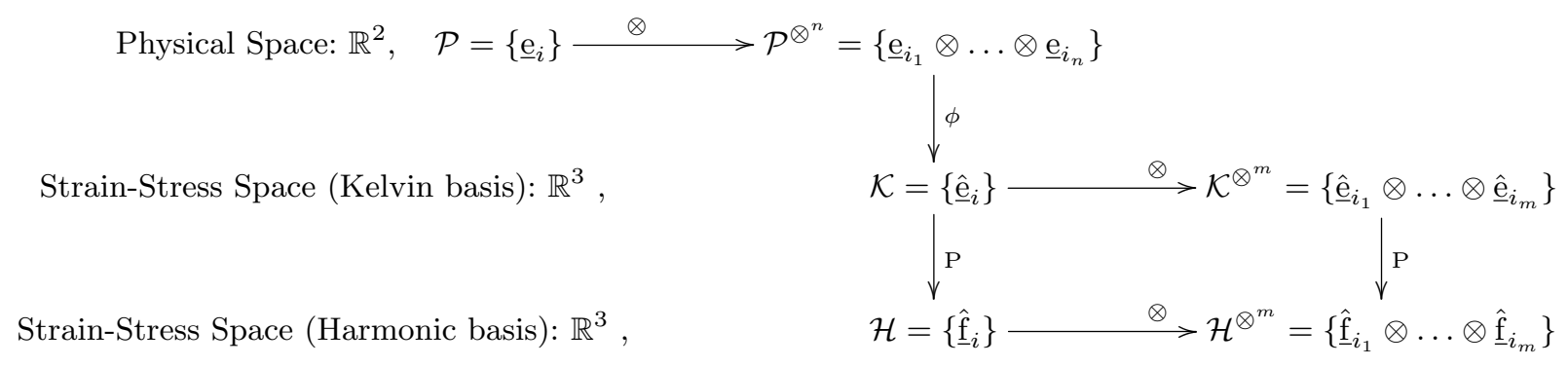


3.2 Representation of physical rotations

The interest of the different bases introduced is revealed when studying how tensors transform with respect to active rotation, that is to element of the rotation subgroup $\mathrm{SO}(2)$. An element of $\mathrm{SO}(2)$ is parametrized by:

$$
\underset{\sim}{\mathrm{R}}=\left(\begin{array}{cc}
\cos \theta & -\sin \theta \\
\sin \theta & \cos \theta
\end{array}\right)_{\mathcal{P}}
$$

Its action on $\underset{\sim}{\mathrm{t}}$ of $S^{2}\left(\mathbb{R}^{2}\right)$ gives a new element $\underset{\sim}{\mathrm{t}}$ of $S^{2}\left(\mathbb{R}^{2}\right)$, the components of which are related to those of $\underset{\sim}{\sim}$ in the following manner

$$
\bar{s}_{i j}=R_{i p} R_{j q} s_{p q}
$$

By defining $\underset{\sim}{\hat{\mathrm{R}}}=\phi(\underset{\sim}{\mathrm{R}} \underset{\sim}{\overline{\mathrm{Q}}})$, the former action expressed in $\mathbb{R}^{2}$, can be reformulated directly in $\widetilde{\mathbb{R}^{3}}$ :

$$
\underline{\hat{\mathrm{t}}}=\underset{\sim}{\hat{\mathrm{R}}} \hat{\mathrm{t}}
$$

In the same way, a rotated elasticity tensor expressed as a second-order tensor in $\mathbb{R}^{3}$ is

$$
\underset{\sim}{\hat{\mathrm{T}}}=\underset{\sim \sim \sim}{\hat{\mathrm{R}}} \hat{\mathrm{R}}^{T}
$$

$\hat{\mathrm{R}}$ has the following matrix expression in $\mathcal{K}$ :

$$
\underset{\sim}{\hat{\mathrm{R}}}=\frac{1}{2}\left(\begin{array}{ccc}
1+\cos 2 \theta & 1-\cos 2 \theta & -\sqrt{2} \sin 2 \theta \\
1-\cos 2 \theta & 1+\cos 2 \theta & \sqrt{2} \sin 2 \theta \\
\sqrt{2} \sin 2 \theta & -\sqrt{2} \sin 2 \theta & 2 \cos 2 \theta
\end{array}\right)_{\mathcal{K}}
$$

It can be checked that $\underset{\sim}{\hat{Q}} \in \mathrm{SO}(3)$ and corresponds to a rotation of $2 \theta$ around the axis $\underline{\mathrm{k}}_{s}$. Expressed in $\mathcal{H}$, the matrix of $\underset{\sim}{\mathrm{Q}}$ has the following expression:

$$
\stackrel{\hat{\mathrm{Q}}}{\sim}=\left(\begin{array}{ccc}
\cos (2 \theta) & -\sin (2 \theta) & 0 \\
\sin (2 \theta) & \cos (2 \theta) & 0 \\
0 & 0 & 1
\end{array}\right)_{\mathcal{H}}
$$

Physical rotation matrices are well-structured in the harmonic basis. The geometric content is clear, when an element of $S^{2}\left(\mathbb{R}^{2}\right)$ is rotated by an angle $\theta$, its spherical part is invariant, while its deviatoric part is turned by an angle $2 \theta$.

\section{Invariant description of $\mathbb{E l a}$}

To construct a geometric description of the space of $2 \mathrm{D}$ elastic materials the use of invariant functions is required. For our needs, those functions will mostly be considered as polynomial. To construct the polynomial invariants a of tensor, the first step is to decomposed this tensor into elementary parts. This decomposition is the object of subsection 4.1. The construction of polynomial invariants and their geometric interpretation will be the object of subsection 4.2 and subsection 4.3. 
4.1 Decompositions of fourth-order elasticity tensors

Let's go back to the study of the space $\mathbb{E}$ la, this space is shown to have the following isotypic structure [3]:

$$
\mathbb{E l a} \simeq \mathbb{K}^{4} \oplus \mathbb{K}^{2} \oplus 2 \mathbb{K}^{0}
$$

in which $\mathbb{K}^{4}$ is the space of $2 \mathrm{D}$ fourth-order harmonic tensors, that is of complete symmetric and traceless fourth-order tensors. Since more than one copy of $\mathbb{K}^{0}$ are involved there are multiple explicit harmonic decompositions [17,3]. This leads to multiple couples of isotropic parameters. A specific choice is the Clebsch-Gordan interpretation of the harmonic decomposition [2]:

$$
\underset{\approx}{\mathrm{T}}=\underset{\approx}{\mathrm{D}}+\frac{1}{2}(\underset{\sim}{1} \otimes \underset{\sim}{\mathrm{d}}+\underset{\sim}{\mathrm{d}} \otimes \underset{\sim}{1})+\kappa(\underset{\sim}{1} \otimes \underset{\sim}{1})+\gamma \underset{\approx}{\mathrm{J}}
$$

where $\underset{\approx}{\mathrm{D}} \in \mathbb{K}^{4}, \mathrm{~d} \in \mathbb{K}^{2}$ and $\{\kappa, \gamma\} \in \mathbb{K}^{0}$.

Using the Kelvin representation a fourth-order elasticity tensor is represented as the following matrix in basis $\mathcal{K}$ :

$$
\hat{\sim}=\left(\begin{array}{ccc}
T_{1111} & T_{1122} & \sqrt{2} T_{1112} \\
T_{1122} & T_{2222} & \sqrt{2} T_{1222} \\
\sqrt{2} T_{1112} & \sqrt{2} T_{1222} & 2 T_{1212}
\end{array}\right)_{\mathcal{K}}
$$

$\underset{\sim}{\mathrm{d}} \in \mathbb{K}^{2}$ and $\underset{\approx}{\mathrm{D}} \in \mathbb{K}^{4}$ can be parametrized in the Kelvin basis by

$$
\underset{\sim}{\hat{\mathrm{d}}}=\left(\begin{array}{c}
d_{1} \\
-d_{1} \\
\sqrt{2} d_{2}
\end{array}\right)_{\mathcal{K}} \quad \stackrel{\hat{\mathrm{D}}}{\sim}=\left(\begin{array}{ccc}
D_{1} & -D_{1} & \sqrt{2} D_{2} \\
-D_{1} & D_{1} & -\sqrt{2} D_{2} \\
\sqrt{2} D_{2} & -\sqrt{2} D_{2} & -2 D_{1}
\end{array}\right)_{\mathcal{K}}
$$

A structure is made apparent by writing the harmonic decomposition of $\mathbb{E}$ la in the $\operatorname{basis}^{2} \mathcal{H}$ :

$$
\hat{\sim}=\left(\begin{array}{ccc}
2 D_{1}+\gamma & 2 D_{2} & d_{1} \\
2 D_{2} & -2 D_{1}+\gamma & d_{2} \\
d_{1} & d_{2} & 2 \kappa
\end{array}\right)_{\mathcal{H}}
$$

The previous matrix is structured which means,

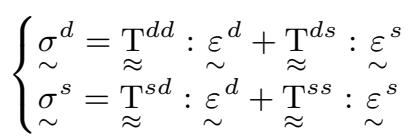

with

$$
\underset{\approx}{\mathrm{T}}=\underset{\approx}{\mathrm{D}}+\gamma \underset{\approx}{\mathrm{J}} \quad ; \quad \underset{\approx}{\mathrm{T}}=\frac{1}{2} \mathrm{~d} \otimes \underset{\sim}{1} \quad ; \quad \underset{\sim}{\mathrm{T}^{s s}}=\kappa(\underset{\sim}{1})
$$

This particular block form for writing the elasticity tensor will be referred to as the Clebsch-Gordan representation. This form consists in writing the elasticity tensor as a symmetric linear application on $\mathbb{K}^{2} \oplus \mathbb{K}^{0}$ :

$$
\mathbb{E l a} \simeq \mathcal{L}^{s}\left(\mathbb{K}^{2} \oplus \mathbb{K}^{0}\right)=\mathcal{L}^{s}\left(\mathbb{K}^{2}\right) \oplus \mathcal{L}^{s}\left(\mathbb{K}^{0}\right) \oplus \mathcal{L}\left(\mathbb{K}^{0}, \mathbb{K}^{2}\right) .
$$

2 The matrix normal forms of elasticity tensor $\underset{\approx}{\mathrm{T}}$ associated to the different symmetry classes are given in the basis $\mathcal{H}$ in Appendix A. 
This decomposition is induced on the linear operator and the elasticity tensor appears to be structured by blocks:

$$
\mathrm{T}=\left[\begin{array}{l:l}
\mathrm{T}^{d d} & \mathrm{~T}^{d s} \\
\hdashline \mathrm{T}^{s d} & \mathrm{~T}^{s} \bar{s} \\
\approx & \approx
\end{array}\right]
$$

4.2 Integrity basis for elasticity tensors

Integrity basis is, for a given space $\mathbb{V}$ and for a given group action $G$, a set of fundamental polynomial invariants such that any $G$-invariant polynomial on $\mathbb{V}$ is a polynomial in the elements of the integrity basis [32]. Integrity basis will be denoted $\mathcal{I B}(\mathbb{V}, G)$.

In the present situation, that is for $\mathbb{V}=\mathbb{E}$ la and $G=\mathrm{O}(2)$, the integrity basis

1. is finitely generated, i.e. $\sharp \mathcal{I} \mathcal{B}(\mathbb{E}$ la, $\mathrm{O}(2))<+\infty$;

2. separates the orbits

$$
\left.\mathcal{I B}(\mathbb{E l a}, \mathrm{O}(2))\left(\underset{\approx}{\mathrm{T}_{1}}\right)=\mathcal{I B}(\mathbb{E l a}, \mathrm{O}(2)) \underset{\approx}{\mathrm{T}_{2}}\right) \Leftrightarrow \underset{\approx}{\mathrm{T}_{1}} \sim \underset{\approx}{\mathrm{T}_{2}} .
$$

In other terms, the invariants of the integrity basis define an application from the space of elasticity tensors to $\mathbb{E}$ la/O(2), the space of elastic materials [5]. From now on, to save space, the notation we will shortened to $\mathcal{I B}$. Integrity bases for $\mathrm{O}(2)$ action on the space of plane elasticity tensors are known since the second-half of the $90[7,31]$ and are constructed from the harmonic decomposition of $\mathbb{E}$ la $[9,21$, $5]$. Let consider the following quantities:

$$
I_{1}=\kappa \quad J_{1}=\gamma \quad I_{2}=\underset{\sim}{\mathrm{d}}: \underset{\sim}{\mathrm{d}} \quad J_{2}=\underset{\approx}{\mathrm{D}}:: \underset{\approx}{\mathrm{D}} \quad I_{3}=\underset{\sim}{\mathrm{d}}: \underset{\approx}{\mathrm{D}}: \underset{\sim}{\mathrm{d}}
$$

Those elements are $\mathrm{O}(2)$-invariant and, in the chosen notation, the subscript indicates the degree of the poynomial invariants in the elasticity tensor.

We have the following result [31]:

Theorem 4.1. A minimal integrity basis for $\mathrm{O}(2)$-action on $\mathbb{E}$ la is

$$
\mathcal{I B}=\left(I_{1}, J_{1}, I_{2}, J_{2}, I_{3}\right) .
$$

Those elements are free meaning that they are not related by any polynomial relation. They satisfy, however, the following inequality:

$$
I_{2}^{2} J_{2}-2 I_{3}^{2} \geq 0
$$

This Cauchy-Schwarz type inequality comes from the fact we are dealing with real valued tensors. This inequality is fundamental and its geometric meaning elucidated in the next subsection.

We define the following application from $\mathbb{E}$ la to $\mathbb{E l a} / \mathrm{O}(2)$ that associate to a tensor its uniquely defined elastic material.

$$
\mathcal{I B}(\underset{\approx}{\mathrm{T}}):=\left(I_{1}(\underset{\approx}{\mathrm{T}}), J_{1}(\underset{\approx}{\mathrm{T}}), I_{2}(\underset{\approx}{\mathrm{T}}), J_{2}(\underset{\approx}{\mathrm{T}}), I_{3}(\underset{\approx}{\mathrm{T}})\right)
$$

This set of polynomial invariants will be referred to as Boehler invariants. 
Remark 4.2. The invariants $I_{2}$ and $J_{2}$ are, by definition, such that

$$
I_{2} \geq 0 \quad J_{2} \geq 0
$$

Remark 4.3. Using the parametrization (8), the invariants $I_{2}, J_{2}, I_{3}$ of the integrity basis read:

$$
I_{2}=2\left(d_{1}^{2}+d_{2}^{2}\right) \quad J_{2}=8\left(D_{1}^{2}+D_{2}^{2}\right) \quad I_{3}=4 D_{1}\left(d_{1}^{2}-d_{2}^{2}\right)+8 D_{2} d_{1} d_{2}
$$

4.3 A geometric representation of the space of elastic materials (vector space $\mathbb{E}$ la)

As introduced in section 2, Ela is divided into 4 strata:

$$
\mathbb{E l a}=\Sigma_{\left[\mathrm{Z}_{2}\right]} \cup \Sigma_{\left[\mathrm{D}_{2}\right]} \cup \Sigma_{\left[\mathrm{D}_{4}\right]} \cup \Sigma_{[\mathrm{O}(2)]}
$$

For the least symmetric class, that is for biclinic elastic materials, the functions of $\mathcal{I B}$ are algebraically independent. A biclinic material is described by five independent quantities, that is by a point in $\mathbb{R}^{5}$. The location of this point is not any, since constrained by the relations (13) and (14).

In Figure 1 are summed-up the different transitions from a symmetry class to another expressed in terms of polynomial relations between invariants. While $\Sigma_{\left[\mathrm{D}_{2}\right]}=\Sigma_{\left[\mathrm{D}_{2}\right]}^{\text {ord }} \cup \Sigma_{\left[\mathrm{D}_{2}\right]}^{s p e c}$, the set $\Sigma_{\left[\mathrm{D}_{2}\right]}^{o r d}$ and $\Sigma_{\left[\mathrm{D}_{2}\right]}^{\text {spec }}$ have been considered in this figure. Elements of the set $\Sigma_{\left[\mathrm{D}_{2}\right]}^{o r d}$ are ordinary orthotropic elasticity tensors. It only contains elements obtained just by imposing orthotropic invariance to generic anisotropic tensors. Elements of the set $\Sigma_{\left[\mathrm{D}_{2}\right]}^{s p e c}$ are special orthotropic tensors, they are not ordinary orthotropic since extra restriction, i.e. other than invariance properties, are needed to define them (those tensors correspond to $R_{0}$-orthotropic tensors [28]).

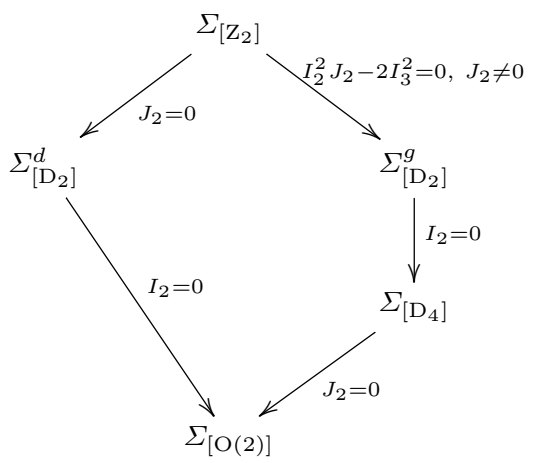

Fig. 1: Breaking symmetry conditions of $\mathbb{E}$ la

In Figure 2 is represented the space of elastic materials (vector space $\mathbb{E}$ la) with respect to $\left(I_{2}, J_{2}, I_{3}\right)$, without taking the positive definiteness property into account. 
The surface, which corresponds to the polynomial equation $I_{2}^{2} J_{2}-2 I_{3}^{2}=0$ contains all the at-least-orthotropic materials (stratum $\overline{\Sigma_{\left[\mathrm{D}_{2}\right]}}=\Sigma_{\left[\mathrm{D}_{2}\right]} \cup \Sigma_{\left[\mathrm{D}_{4}\right]} \cup \Sigma_{[\mathrm{O}(2)]}$ ). The condition $I_{2}^{2} J_{2}-2 I_{3}^{2}>0$ indicates on which side of the orthotropic surface are the biclinic materials located (strata $\Sigma_{\left[\mathrm{Z}_{2}\right]}$ ). Finally, we get that, independently of the values of the isotropic invariants $I_{1}$ and $J_{1}$ :

- biclinic materials (stratum $\Sigma_{\left[\mathrm{Z}_{2}\right]}$ ) are strictly inside the volume defined by the surface,

- point $O$ corresponds to isotropic materials (stratum $\left.\Sigma_{[\mathrm{O}(2)]}\right)$,

- open ray $] O A$ ) corresponds to tetragonal materials (stratum $\Sigma_{\left[\mathrm{D}_{4}\right]}$ ),

- open ray $] O B$ ) corresponds to $R_{0}$-orthotropic materials (stratum $\Sigma_{\left[\mathrm{D}_{2}\right]}^{s p e c}$ ),

- surface without $\{O\} \cup] O A) \cup] O B$ ) corresponds to ordinary orthotropic materials (stratum $\Sigma_{\left[\mathrm{D}_{2}\right]}^{o r d}$ ).

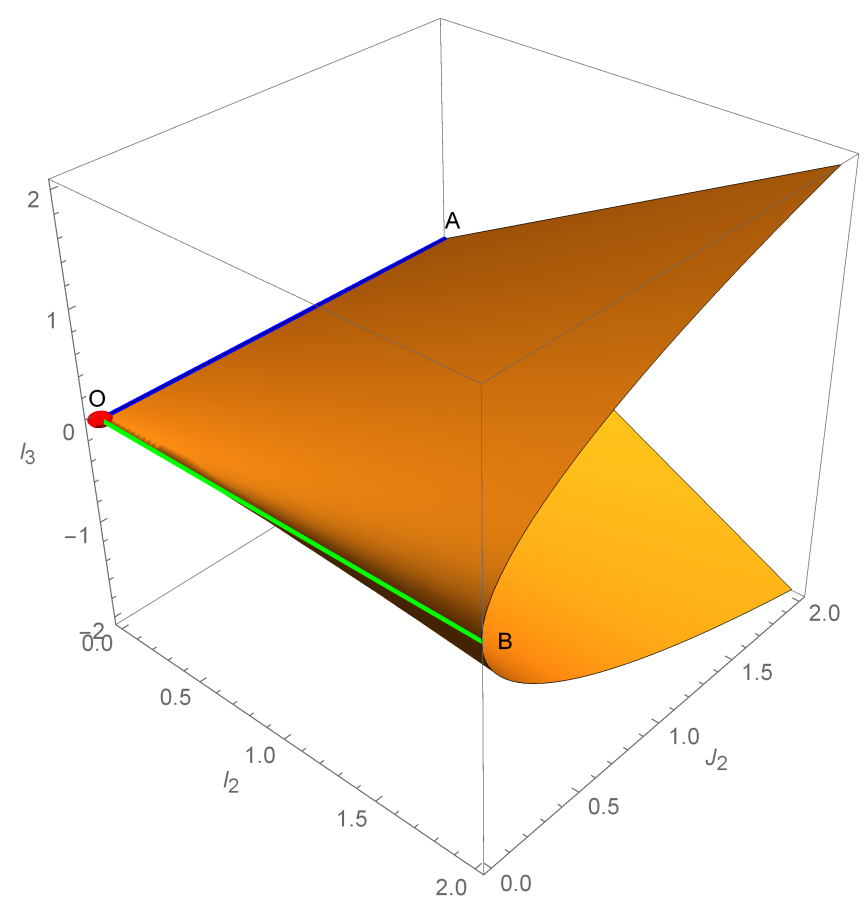

Fig. 2: Algebraic variety of elastic material with respect to $\left(I_{2}, J_{2}, I_{3}\right)$ (without taking the positive definiteness property into account).

\section{Invariant based positive definiteness condition for elastic materials}

As previously said in section 2.1 , an element $\underset{\approx}{\mathrm{T}}$ of $\mathbb{E}$ la is admissible if, considered as a linear application on $S^{2}\left(\mathbb{R}^{2}\right)$, its eigenvalues $\lambda_{i}$ verifies

$$
\exists M \in \mathbb{R}^{*+}, \quad 0<\lambda_{i} \leq M
$$


so that only the restriction of $\mathbb{E}$ la to the cone ${ }^{3}$ of symmetric definite elasticity tensors can be associated to elastic materials. Our aim is now to express this condition using the invariants of the integrity basis $\mathcal{I B}$.

First, it has to be observed that the eigenvalues of $\underset{\approx}{\mathrm{T}}$ are algebraic (i.e. roots of a polynomial equation) invariants of $\mathrm{O}(3)$. We have the following lemma [23]:

Lemma 5.1. A symmetric matrix $M$ in $\mathcal{M}_{n}(\mathbb{R})$ is positive definite if and only if $\sigma_{k}(M)>0$, with $\left\{\sigma_{k}\right\}$ the set of elementary symmetric polynomials.

It can be checked by a direct calculation that

$$
\left\{\begin{array}{l}
\sigma_{1}=2\left(I_{1}+J_{1}\right) \\
\sigma_{2}=4 I_{1} J_{1}+J_{1}^{2}-\frac{1}{2}\left(I_{2}+J_{2}\right) \\
\sigma_{3}=\frac{1}{2}\left(I_{3}-I_{2} J_{1}+4 I_{1} J_{1}^{2}-2 I_{1} J_{2}\right)
\end{array}\right.
$$

so that the the positivity conditions can be expressed in terms of elements of the integrity basis:

$$
\underset{\approx}{\mathrm{T}} \text { is positive definite } \Leftrightarrow\left\{\begin{array}{l}
I_{1}+J_{1}>0 \\
4 I_{1} J_{1}+J_{1}^{2}-\frac{1}{2}\left(I_{2}+J_{2}\right)>0 \\
I_{3}-I_{2} J_{1}+4 I_{1} J_{1}^{2}-2 I_{1} J_{2}>0
\end{array}\right.
$$

To obtain simpler expressions, the Clebsch-Gordan form of the elasticity tensor can be exploited. To that aim consider the following lemma [23]:

Lemma 5.2. Consider $M \in \mathcal{M}_{p+q}^{S}$ a real symmetric matrix having the following block shape

$$
M=\left(\begin{array}{cc}
A & B \\
B^{T} & C
\end{array}\right)
$$

with $A \in \mathcal{M}_{p}^{S}, C \in \mathcal{M}_{q}^{S}, B \in \mathcal{M}_{p, q}$. Let $M / A$ be the Schur complement of $A$ in $M$ :

$$
M / A=C-B^{T} A^{-1} B
$$

and $M / C$ be the Schur complement of $C$ in $M$ :

$$
M / C=A-B C^{-1} B^{T}
$$

Then the following conditions are equivalent:

1. $M$ is positive definite if and only if $A$ and $M / A$ are positive definite,

2. $M$ is positive definite if and only if $C$ and $C / A$ are positive definite.

\footnotetext{
${ }^{3}$ A subset $\mathcal{C}$ of a vector space $\mathbb{V}$ is a cone if for each $p$ in $\mathcal{C}$ and positive scalars $\lambda, \lambda p \in \mathcal{C}$. The cone is said convex provided $\lambda p+\mu q \in \mathcal{C}$, for any positive scalars $\lambda, \mu$ and any $p, q \in \mathcal{C}$
} 
This approach applied respectively to $\underset{\approx}{\mathrm{T}} d d$ and to $\underset{\approx}{\mathrm{T}}{ }^{s s}$ (defined in (10)) provides the following equivalent sets of positiveness conditions expressed in terms of the elements of the integrity basis:

$$
\begin{aligned}
\underset{\mathrm{T}}{\approx} \text { is positive definite } & \Leftrightarrow\left\{\begin{array}{l}
J_{1}>0 \\
2 J_{1}^{2}-J_{2}>0 \\
I_{3}-2 J_{2} I_{1}-I_{2} J_{1}+4 J_{1}^{2} I_{1}>0
\end{array}\right. \\
& \Leftrightarrow\left\{\begin{array}{l}
I_{1}>0 \\
8 I_{1} J_{1}-I_{2}>0 \\
I_{3}-2 J_{2} I_{1}-I_{2} J_{1}+4 J_{1}^{2} I_{1}>0
\end{array}\right.
\end{aligned}
$$

The positiveness conditions show that for any elastic material, the isotropic invariants $I_{1}$ and $J_{1}$ are strictly positive quantities. A look at the two sets of conditions suggests to introduce the following adimensional anisotropic invariants:

$$
i_{2}=\frac{I_{2}}{8 I_{1} J_{1}} \quad j_{2}=\frac{J_{2}}{2 J_{1}^{2}} \quad i_{3}=\frac{I_{3}}{8 I_{1} J_{1}^{2}}
$$

The positive definiteness conditions read

$$
\underset{\approx}{\mathrm{T}} \text { is positive definite } \Leftrightarrow\left\{\begin{array} { l } 
{ 0 \leq j _ { 2 } < 1 } \\
{ 2 i _ { 3 } - j _ { 2 } - 2 i _ { 2 } + 1 > 0 }
\end{array} \Leftrightarrow \left\{\begin{array}{l}
0 \leq i_{2}<1 \\
2 i_{3}-j_{2}-2 i_{2}+1>0
\end{array}\right.\right.
$$

The adimensionalization of the inequality (13) shows that

$$
-1<i_{3}<+1
$$

Together with condition (13), the complete set ${ }^{4}$ of conditions for positive definiteness of a real valued fourth-order elasticity tensor read

$$
\left\{\begin{array}{l}
0 \leq i_{2}<1 \\
0 \leq j_{2}<1 \\
-1 \leq i_{3} \leq+1 \\
\frac{1}{2}\left(-1+2 i_{2}+j_{2}\right)<i_{3} \\
i_{3}^{2} \leq i_{2}^{2} j_{2}
\end{array}\right.
$$

Such a domain is presented in figure 3. Note that the quantities on the three axes are different from figure 2. The geometric domain of positive definite elasticity tensors is strictly located above the plane and inside the volume defined by the surface. For $j_{2}=1$, the plane and the surface are coincident.

\footnotetext{
4 The number of inequations in the presented set is not minimal.
} 

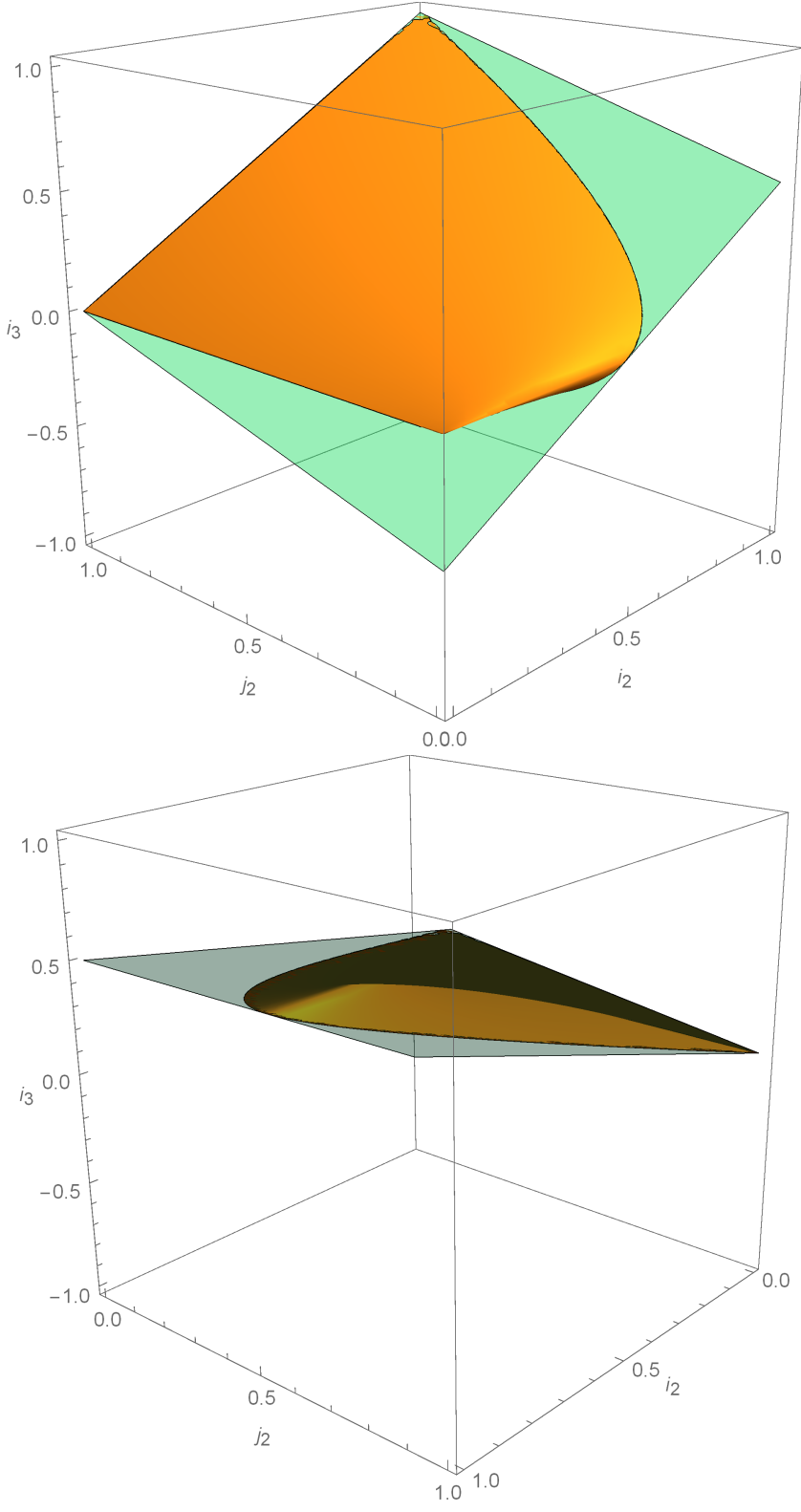

Fig. 3: Positive definiteness elasticity domain in terms of adimensional anisotropic invariants (front and back views).

Remark 5.3. By doing the normalization procedure (18), the set of adminensional invariants does not design a unique elastic material anymore but rather a family of elastic materials. It is possible to define the following equivalence relation, two 
elastic tensors $\underset{\approx}{\mathrm{T}_{1}}, \underset{\approx}{\mathrm{T}_{2}} \in \mathbb{E}$ la are equivalent if and only if

$$
\exists \alpha, \beta \in \mathbb{R},(\alpha, \beta) \neq(0,0) \text { such that } \underset{\sim}{\hat{\mathrm{T}}}=\underset{\sim}{\mathrm{H}}(\alpha, \beta) \underset{\sim}{\hat{\mathrm{T}}} \underset{\sim}{\mathrm{H}}(\alpha, \beta)^{T}
$$

with $^{5}$

$$
\underset{\sim}{\mathrm{H}}(\alpha, \beta)=\left(\begin{array}{lll}
\alpha & 0 & 0 \\
0 & \alpha & 0 \\
0 & 0 & \beta
\end{array}\right)_{\mathcal{H}}
$$

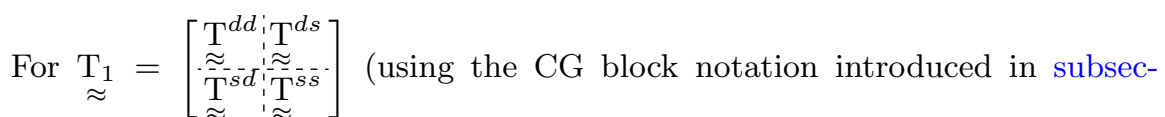
tion 4.1), elasticity tensors of the following form

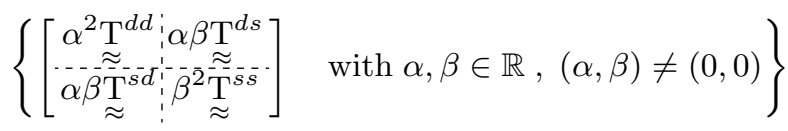

possess identical adimensional invariants. More details can be found in Appendix D.

\section{An explicit link between Boehler and Kelvin invariants}

The invariants considered in this paper are polynomial functions of the tensor considered as a geometric object in the physical space $\mathbb{R}^{2}$, the group of invariance being $\mathrm{O}(2)$. Hereafter these quantities will be referred to as the Boehler invariants $[9,5]$. The eigenvalues of symmetric tensors constitute another system of invariant functions. Since eigenvalues are algebraic invariants, we will rather consider the coefficients of the characteristic polynomial which are given by the elementary symmetric polynomials. Those quantities will be referred to as (polynomial) Kelvin invariants. The two sets (with Boehler and Kelvin invariants) coincide only for second-order tensors. For the space of fourth-order elasticity tensors Ela, the Boelher set comprises 5 invariants $\left(I_{1}, J_{1}, I_{2}, J_{2}, I_{3}\right)$, while Kelvin set comprises only 3 quantities $\left(\sigma_{1}, \sigma_{2}, \sigma_{3}\right)$. To build a bridge between these sets for elasticity tensors, let consider the following classical result $[24,26,8,33]$ :

Lemma 6.1. Let $(\underset{\sim}{\mathrm{a}}, \underline{\mathrm{u}}) \in \mathbb{V}=S^{2}\left(\mathbb{R}^{3}\right) \times \mathbb{R}^{3}$ and set:

$$
\begin{array}{lll}
i_{1,0}:=\operatorname{tr} \underset{\sim}{\mathrm{a}}, & i_{2,0}:=\operatorname{tr}{\underset{\sim}{\mathrm{a}}}^{2}, & i_{3,0}:=\operatorname{tr} \underset{\sim}{\sim} \stackrel{\sim}{\sim}^{3}, \\
i_{0,2}:=\underline{\mathrm{u}} \cdot \underline{\mathrm{u}}, & i_{1,2}:=\underline{\mathrm{u}} \cdot(\underset{\mathrm{a}}{\sim} \cdot \underline{\mathrm{u}}), & i_{2,2}:=\underline{\mathrm{u}} \cdot\left({\underset{\sim}{\mathrm{a}}}^{2} \cdot \underline{\mathrm{u}}\right),
\end{array}
$$

A minimal integrity basis $\mathcal{I B}$ for $\mathbb{V}$ with respect to standard $\mathrm{O}(3)$-action, is given by the collection:

$$
\mathcal{I B}(\mathbb{V})=\left\{i_{1,0}, i_{2,0}, i_{3,0}, i_{0,2}, i_{1,2}, i_{2,2}\right\}
$$

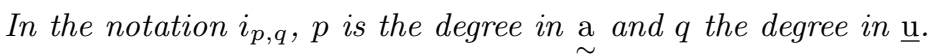

\footnotetext{
5 The matrices $\underset{\sim}{\mathrm{H}}(\alpha, \beta)$ and $\underset{\sim}{\hat{\mathrm{R}}}(\theta)$ commute.
} 
Remark 6.2. For $\underset{\sim}{\mathrm{a}} \in S^{2}\left(\mathbb{R}^{3}\right)$, the Newton sums $\operatorname{tr} \underset{\sim}{\mathrm{a}}, \operatorname{tr} \underset{\sim}{\mathrm{a}}{ }^{2}$ and $\operatorname{tr} \underset{\sim}{\mathrm{a}}{ }^{3}$ are related to the elementary symmetric functions $\sigma_{1}, \sigma_{2}, \sigma_{3}$ by the uniquely invertible following relations:

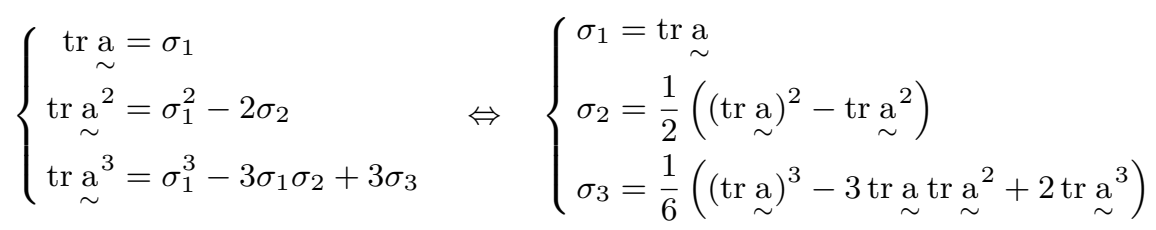

This allows to exchange the first 3 invariants $i_{1,0}, i_{2,0}, i_{3,0}$ in (20) of lemma 6.1 with the elementary symmetric functions.

Proposition 6.3. Consider $\underset{\approx}{\mathrm{T}} \in \mathbb{E}$ la and $\underset{\sim}{\hat{\mathrm{T}}}$ its representation as a second-order tensor in $\mathbb{R}^{3}$. Consider also the tensor

$$
\underset{\sim}{\mathrm{s}}=\frac{\sqrt{2}}{2}\left(\underline{\mathrm{e}}_{1} \otimes \underline{\mathrm{e}}_{1}+\underline{\mathrm{e}}_{2} \otimes \underline{\mathrm{e}}_{2}\right)
$$

and $\underline{\hat{\mathrm{s}}}$ its vector representation in $\mathbb{R}^{3}$. The set of Boehler invariants of $\underset{\approx}{\mathrm{T}}$ for the $\mathrm{O}(2)$-action is equivalent to the set of invariants of the pair $(\underset{\sim}{\hat{\mathrm{T}}}, \underline{\hat{\mathrm{s}}})$ for the $\mathrm{O}(3)$ action.

Proof. The vector $\underline{\hat{\mathrm{s}}}$ is unitary, hence $i_{0,2}=1$ and no specific information is given by this quantity. The following relations can be verified by a direct calculation:

$$
\left\{\begin{array}{l}
\sigma_{1}=2\left(I_{1}+J_{1}\right) \\
\sigma_{2}=4 I_{1} J_{1}+J_{1}^{2}-\frac{1}{2}\left(I_{2}+J_{2}\right) \\
\sigma_{3}=\frac{1}{2}\left(I_{3}-I_{2} J_{1}+4 I_{1} J_{1}^{2}-2 I_{1} J_{2}\right) \\
i_{1,2}=2 I_{1} \\
i_{2,2}=\frac{1}{2} I_{2}+4 I_{1}^{2}
\end{array}\right.
$$

This non-linear system is easily uniquely inverted by substitution in the following:

$$
\left\{\begin{array}{l}
I_{1}=\frac{1}{2} i_{1,2} \\
J_{1}=\frac{1}{2}\left(\sigma_{1}-i_{1,2}\right) \\
I_{2}=2\left(i_{2,2}-i_{1,2}^{2}\right) \\
J_{2}=\frac{1}{2}\left(\sigma_{1}+i_{1,2}\right)^{2}-2\left(\sigma_{2}+i_{2,2}\right) \\
I_{3}=2 \sigma_{3}-2 \sigma_{2} i_{1,2}+i_{1,2}^{2}\left(\sigma_{1}+i_{1,2}\right)+i_{2,2}\left(\sigma_{1}-3 i_{1,2}\right)
\end{array}\right.
$$

Again, this system is uniquely invertible (by substitution). This ends the proof.

This result shows that Kelvin invariants is the subset of Boehler invariants obtained when the spheric direction $\underline{\hat{s}}$ is forgot. As a consequence the eigenvalues of the elasticity tensor do not define uniquely an elastic material, but rather a family of elastic materials.

Using the spheric direction $\underline{\hat{s}}$, it is possible to give the following alternative definition of Boehler invariants. Consider $\underline{\hat{s}}$ the vector direction of the spheric space in $\mathbb{R}^{3}$ and construct the projectors 


$$
\stackrel{\mathrm{P}}{s}^{s}=\underline{\hat{\mathrm{s}}} \otimes \underline{\hat{\mathrm{s}}} \quad ; \quad \stackrel{\mathrm{P}}{ }^{d}=\underset{\sim}{1}-\underline{\mathrm{P}}^{s}
$$

The Clesbch-Gordan elements of the harmonic decomposition can be defined as follows:

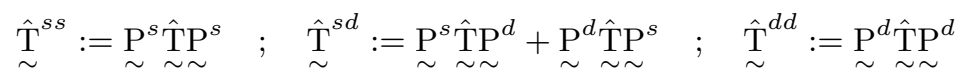

The Boehler invariants of the integrity basis are:

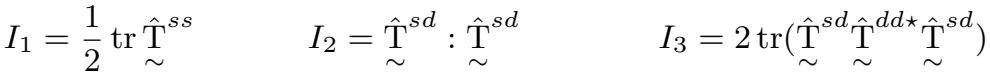

$$
\begin{aligned}
& J_{1}=\frac{1}{2} \operatorname{tr}{\underset{\sim}{\hat{T}}}^{d d} \quad J_{2}={\underset{\sim}{\hat{T}}}^{d d \star}:{\underset{\sim}{T}}^{d d \star}
\end{aligned}
$$

in which the notation $\star$ is defined by

$$
\underset{\sim}{\mathrm{T}^{\star}}:=\underset{\sim}{\mathrm{T}}-\frac{1}{2}(\operatorname{tr} \underset{\sim}{\mathrm{T}}) \mathrm{P}_{\sim}^{d}
$$

\section{Design transformations}

Having two different actions acting on the space of elasticity tensors motivates the introduction of a new concept that we called design transformation.

First, the design transformation is defined in section 7.1. Then, we will prove in section 7.2 that any elastic material can be design-transformed into a tetragonal elastic material. Finally, we will study in details in section 7.3 the design transformations of initially isotropic elastic materials.

\subsection{Definition and fundamental properties of a design transformation}

Definition 7.1. Applications between elastic materials preserving eigenvalues will be called design transformations.

The design transformations have the following fundamental properties:

1. the set of design transformations of $\mathbb{E}$ la is $\mathrm{SO}(3)$;

2. any initially positive definite tensor remains positive definite after any designtransformation;

3. trivial design transformations are the physical rotations.

\subsection{Tetragonal equivalent elastic material}

We have the following remarkable property:

Proposition 7.2. Except for isotropic elasticity tensors with 3 identical eigenvalues, any elasticity tensor $\underset{\approx}{\mathrm{T}} \in \mathbb{E}$ la is equivalent, up to a design transformation, to at least one tetragonal elasticity tensor. 
Proof. Consider an elasticity tensor $\underset{\approx}{\mathrm{T}} \in \mathbb{E}$ la and $\underset{\sim}{\hat{\mathrm{T}}}$ its second-order tensor representation in $S^{2}\left(\mathbb{R}^{3}\right)$. Consider $[\underset{\sim}{\hat{T}}]_{\mathcal{H}}$, the matrix representation of $\underset{\sim}{\hat{T}}$ in the harmonic basis $\mathcal{H}$. Diagonalization of $[\underset{\sim}{\hat{T}}]_{\mathcal{H}}$ allows for defining a rotation which ends up with a diagonal matrix in basis $\mathcal{H}$. Let denote $\left\{\lambda_{i}\right\}_{1 \leq i \leq 3}$ the set of eigenvalues. Suppose first that the $\left\{\lambda_{i}\right\}_{1 \leq i \leq 3}$ are all distinct (this is the standard situation for a initial tensor that belongs to $\Sigma_{\left[\mathrm{D}_{4}\right]}, \Sigma_{\left[\mathrm{D}_{2}\right]}$ or $\left.\Sigma_{\left[\mathrm{Z}_{2}\right]}\right)$. In this case there are 6 seemingly different permutations of this set. It can be observed that a $\frac{\pi}{2}$ physical rotation will permute the first two eigenvalues. Hence we end up with 3 distinct $\Sigma_{\left[\mathrm{D}_{4}\right]}$ materials. Suppose now that among $\left\{\lambda_{i}\right\}_{1 \leq i \leq 3}$ two eigenvalues are equal. Let say that we have $\left\{\lambda_{1}, \lambda_{1}, \lambda_{2}\right\}$ (this is the standard situation for a initial tensor that belongs to $\Sigma_{[\mathrm{O}(2)]}$ and the exceptional situation for tensor in $\Sigma_{\left[\mathrm{D}_{4}\right]}$ or $\left.\Sigma_{\left[\mathrm{D}_{2}\right]}\right)$. In this case there are 3 seemingly different permutations of this set. It can be observed that a $\frac{\pi}{2}$ physical rotation will permute the first two eigenvalues. Hence we end up with 2 distinct situations : $\left\{\lambda_{1}, \lambda_{1}, \lambda_{2}\right\}$ and $\left\{\lambda_{1}, \lambda_{2}, \lambda_{1}\right\}$. The first one correspond to a tensor in $\Sigma_{[\mathrm{O}(2)]}$ tensor, while the other to a tensor in $\Sigma_{\left[\mathrm{D}_{4}\right]}$.

7.3 Study of design-transformed initially isotropic material

Let consider an isotropic elastic material:

$$
\hat{\mathrm{T}}=\left(\begin{array}{ccc}
\gamma_{0} & 0 & 0 \\
0 & \gamma_{0} & 0 \\
0 & 0 & 2 \kappa_{0}
\end{array}\right)_{\mathcal{H}}
$$

Using a unit quaternion, any rotation in $\mathrm{SO}(3)$ can be encoded as (see appendix B)

$$
\underset{\mathrm{Q}}{\sim}=\left(\begin{array}{ccc}
\omega^{2}+v_{x}^{2}-v_{y}^{2}-v_{z}^{2} & 2\left(v_{x} v_{y}-\omega v_{z}\right) & 2\left(\omega v_{y}+v_{x} v_{z}\right) \\
2\left(v_{x} v_{y}+\omega v_{z}\right) & \omega^{2}-v_{x}^{2}+v_{y}^{2}-v_{z}^{2} & 2\left(v_{y} v_{z}-\omega v_{x}\right) \\
2\left(v_{x} v_{z}-\omega v_{y}\right) & 2\left(\omega v_{x}+v_{y} v_{z}\right) & \left(\omega^{2}-v_{x}^{2}-v_{y}^{2}+v_{z}^{2}\right)
\end{array}\right)
$$

with

$$
\omega^{2}+v_{x}^{2}+v_{y}^{2}+v_{z}^{2}=1
$$

Let introduce the parameter $\alpha \in[0,1]$ defined by

$$
\alpha:=v_{x}^{2}+v_{y}^{2} \text {. }
$$

Using equation (15) and property $\alpha=1-v_{z}^{2}-\omega^{2}$, the family of elastic materials obtained by carrying out design transformations on the initially isotropic elastic material (21) is obtained as a parametric set of the following form, with $\alpha \in[0,1]$ :

$$
\mathcal{I B}(\alpha)=\left\{\begin{array}{l}
I_{1}(\alpha)=\kappa_{0}-2 \alpha(1-\alpha)\left(2 \kappa_{0}-\gamma_{0}\right) \\
J_{1}(\alpha)=\gamma_{0}+2 \alpha(1-\alpha)\left(2 \kappa_{0}-\gamma_{0}\right) \\
I_{2}(\alpha)=8 \alpha(1-\alpha)(1-2 \alpha)^{2}\left(2 \kappa_{0}-\gamma_{0}\right)^{2} \\
J_{2}(\alpha)=8 \alpha^{2}(1-\alpha)^{2}\left(2 \kappa_{0}-\gamma_{0}\right)^{2} \\
I_{3}(\alpha)=16 \alpha^{2}(1-\alpha)^{2}(1-2 \alpha)^{2}\left(2 \kappa_{0}-\gamma_{0}\right)^{3}
\end{array}\right.
$$

It can be noticed that: 
- $\mathcal{I B}(\alpha)=\mathcal{I B}(1-\alpha) \quad \forall \alpha \in[0,1]$ so that the variation of parametric parameter $\alpha$ can be reduced to the interval $\left[0, \frac{1}{2}\right]$;

- for $\alpha=0$ the initial isotropic material is retrieved;

- for $\alpha=\frac{1}{2}$ a tetragonal material is obtained;

- $I_{1}(\alpha)+J_{1}(\alpha)=\kappa_{0}+\gamma_{0}, \forall \alpha \in\left[0, \frac{1}{2}\right]$ so that the curve in the $\left(I_{1}, J_{1}\right)$ plane is a line segment;

$-2 I_{3}(\alpha)^{3}-I_{2}(\alpha)^{2} J_{2}(\alpha)=0, \forall \alpha \in\left[0, \frac{1}{2}\right]$ so that the curve is drawn on the surface of orthotropic elastic materials.

In other terms, the last item indicates that no biclinic material can be reached by the design transformation of an initially isotropic material.

The parametric set of equations (23) describe a curve in the space of elastic materials. This curve is represented in figure 4 for specific values of $\kappa_{0}$ and $\gamma_{0}$ (as the material remains positive definite, the positive definiteness condition is not plotted on the graph).
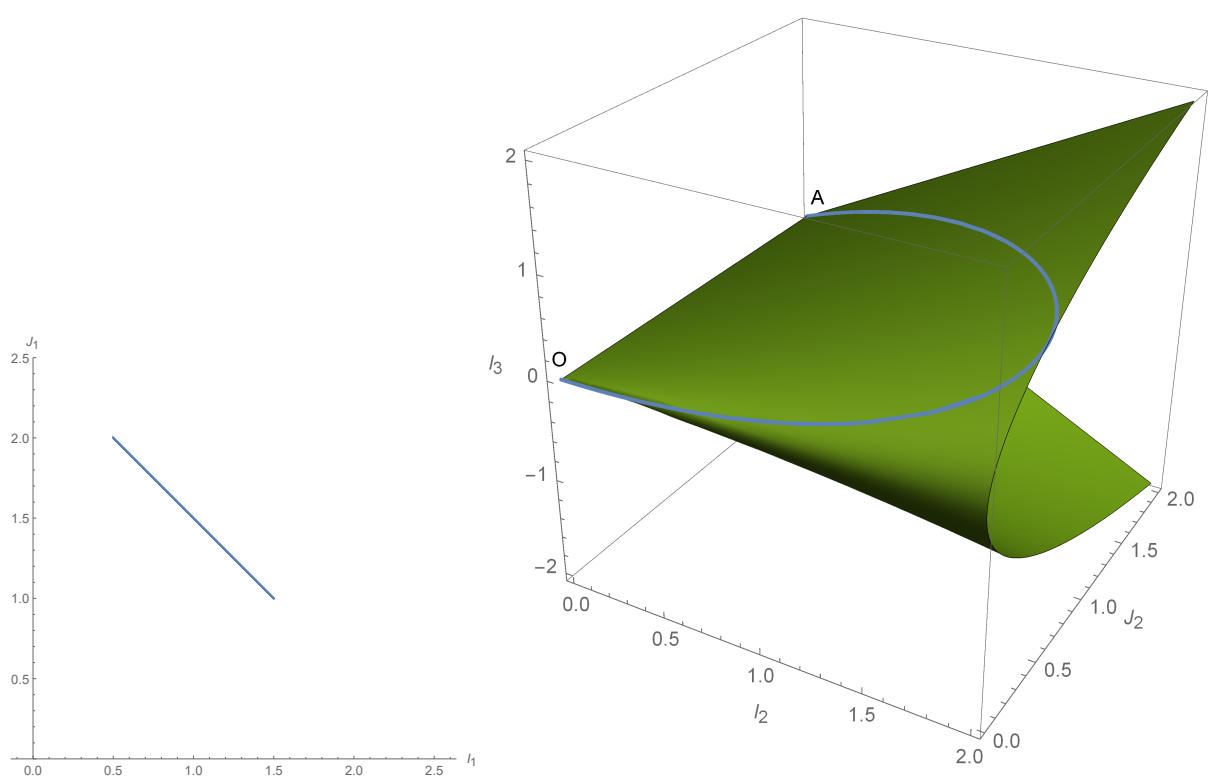

Fig. 4: An iso-eigenvalues curve in the space of elastic material. The initial material is isotropic (point $\mathrm{O}, \alpha=0$ ), with the design transformation it becomes orthotropic (curved line, $0<\alpha<\frac{1}{2}$ ) and becomes tetragonal for $\alpha=\frac{1}{2}$ (point A). Figure plotted with $\gamma_{0}=1$ and $\kappa_{0}=\frac{3}{2}$.

Remark 7.3. The characterization in terms of axes and angle of rotations with a fixed value of $\alpha$ is given in appendix B. Note that the entire curve can be obtained by considering a rotation of any axe orthogonal to $\underline{\hat{f}}_{3}$ and angle varying in $[0, \pi]$. 
The variable $\alpha$ can be eliminated from (23) leading to the following equivalent system of equations, where $I_{1} \in\left[\frac{\gamma_{0}}{2}, \kappa_{0}\right]$ :

$$
\left\{\begin{array}{l}
J_{1}=\gamma_{0}+\kappa_{0}-I_{1} \\
I_{2}=4\left(2 I_{1}-\gamma_{0}\right)\left(\kappa_{0}-I_{1}\right) \\
J_{2}=2\left(\kappa_{0}-I_{1}\right)^{2} \\
I_{3}=4\left(2 I_{1}-\gamma_{0}\right)\left(\kappa_{0}-I_{1}\right)^{2}
\end{array}\right.
$$

Moreover, the variables $\left(\gamma_{0}, \kappa_{0}\right)$ can be eliminated from the previous system, leading to the following implicit algebraic system of equations, where $I_{1}>0$ and $J_{1}>0$ :

$$
\left\{\begin{array}{l}
I_{2}^{2}-32 I_{1}^{2} J_{2}-4 I_{2} J_{2}+32 I_{1} J_{1} J_{2}-8 J_{1}^{2} J_{2}+4 J_{2}^{2}=0 \\
I_{2}^{2}-8 I_{1} I_{3}+4 I_{3} J_{1}-2 I_{2} J_{2}=0 \\
-I_{2} I_{3}+4 I_{1} I_{2} J_{2}+2 I_{3} J_{2}-2 I_{2} J_{1} J_{2}=0 \\
2 I_{3}^{2}-I_{2}^{2} J_{2}=0
\end{array}\right.
$$

The algebraic system of equations (24) characterizes the set of at-least-orthotropic elastic materials that can be design-transformed into an isotropic material. Moreover, we have the following property (proof given in Appendix C):

Proposition 7.4. The algebraic system (24) corresponds exactly to the subset of at-least-orthotropic materials with two identical eigenvalues.

7.4 Particular case of pentamode materials

Let consider an isotropic elastic material:

$$
\stackrel{\hat{T}}{\sim}_{0}=\left(\begin{array}{ccc}
0 & 0 & 0 \\
0 & 0 & 0 \\
0 & 0 & 2 \kappa_{0}
\end{array}\right)_{\mathcal{H}}
$$

The integrity basis reads then

$$
\mathcal{I} \mathcal{B}(\alpha)=\left\{\begin{array}{l}
I_{1}(\alpha)=(1-2 \alpha)^{2} \kappa_{0} \\
J_{1}(\alpha)=4 \alpha(1-\alpha) \kappa_{0} \\
I_{2}(\alpha)=32 \alpha(1-\alpha)(1-2 \alpha)^{2} \kappa_{0}^{2} \\
J_{2}(\alpha)=32 \alpha^{2}(1-\alpha)^{2} \kappa_{0}^{2} \\
I_{3}(\alpha)=128 \alpha^{2}(1-\alpha)^{2}(1-2 \alpha)^{2} \kappa_{0}^{3}
\end{array} \quad, \alpha \in\left[0, \frac{1}{2}\right]\right.
$$

When the variable $\alpha$ is eliminated, this leads to the following equivalent system of equations, where $I_{1} \in\left[0, \kappa_{0}\right]$ :

$$
\left\{\begin{array}{l}
J_{1}=\kappa_{0}-I_{1} \\
I_{2}=8 I_{1}\left(\kappa_{0}-I_{1}\right) \\
J_{2}=2\left(\kappa_{0}-I_{1}\right)^{2} \\
I_{3}=8 I_{1}\left(\kappa_{0}-I_{1}\right)^{2}
\end{array}\right.
$$


The parameter $\kappa_{0}$ can be eliminated from the previous system of equation, where $I_{1} \geq 0$ and $J_{1} \geq 0$ :

$$
\left\{\begin{array}{l}
I_{2}=8 I_{1} J_{1} \\
J_{2}=2 J_{1}^{2} \\
I_{3}=8 I_{1} J_{1}^{2}
\end{array}\right.
$$

(26) is an algebraic system of equations that defines the entire set of at-leastorthotropic elastic materials that can be design-transformed into an isotropic material with two eigenvalues equal to zero. Moreover, we have the following property (proof given in Appendix C):

Proposition 7.5. The algebraic system (26) corresponds to the set of at-leastorthotropic materials with 2 eigenvalues equal to zero.

\section{A Normal forms}

In this appendix, normal forms for tensors of different symmetry classes are provided with respect to the harmonic basis ${ }^{6} \mathcal{H}$. Normal forms are provided for generic tensors within each symmetry class. In the following, $\bullet$ corresponds to independent coefficients while $\times$ indicates equal coefficients.

$-\Sigma_{\left[Z_{2}\right]}$
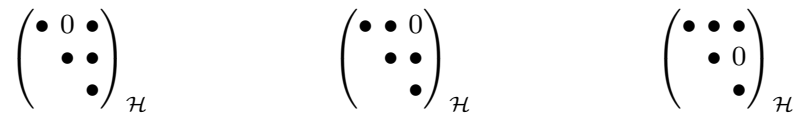

Remark: A physical rotation of $\frac{\pi}{2}$ switches normal form 2 to normal form 3.

$-\Sigma_{\left[\mathrm{D}_{2}\right]}$

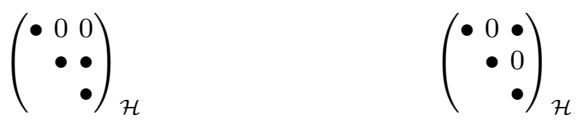

Remark: A physical rotation of $\frac{\pi}{2}$ switches the normal form one to another.

$-\Sigma_{\left[\mathrm{D}_{4}\right]}$

$$
\left(\begin{array}{rrr}
\bullet & 0 & 0 \\
\bullet & 0 \\
& \bullet
\end{array}\right)_{\mathcal{H}}
$$

$-\Sigma_{[\mathrm{O}(2)]}$

$$
\left(\begin{array}{ccc}
\times & 0 & 0 \\
& \times & 0 \\
& & \bullet
\end{array}\right)_{\mathcal{H}}
$$

\section{B Quaternionic parametrization of rotations}

Let denote $\mathcal{S}^{d}$ the unit sphere in dimension $d$. Consider $q$ a pure quaternion:

$$
q \in \operatorname{Im}(\mathbb{H}) \Leftrightarrow q=q_{x} \mathbf{i}+q_{y} \mathbf{j}+q_{z} \mathbf{k}
$$

6 It is worth emphasizing that usually normal forms are provided with respect to the Voigt or the Kelvin representation. 
with the Hamilton relations

$$
\mathbf{i}^{2}=\mathbf{j}^{2}=\mathbf{k}^{2}=\mathbf{i j k}=-1
$$

As $q \in \operatorname{Im}(\mathbb{H}), q$ will be denoted by $\underline{q}$ from now on.

$\mathrm{SO}(3)$ action on $\operatorname{Im}(\mathbb{H})$ is

$$
\underline{\mathrm{q}}^{\star}=h \underline{\mathrm{q}}^{-1}
$$

with $h \in \mathcal{S}^{3}$ the space of unit quaternions.

$$
h \in \mathcal{S}^{3} \Leftrightarrow h=\omega+v_{x} \mathbf{i}+v_{y} \mathbf{j}+v_{z} \mathbf{k} \quad \text { with } \quad \omega^{2}+v_{x}^{2}+v_{y}^{2}+v_{z}^{2}=1
$$

As a quaternion the expression of $\mathrm{h}$ can be shortened to

$$
h=\omega+\underline{\mathrm{v}}
$$

in which $\omega$ is the scalar/real part of $h$ and $\underline{\mathrm{v}}$ is the vector/imaginary part of $h$. Unit quaternions can also be expressed under the so called polar form:

$$
h=\cos \left(\frac{\theta}{2}\right)+\underline{\mathrm{n}} \sin \left(\frac{\theta}{2}\right) \quad \text { with } \underline{\mathrm{n}} \in \mathcal{S}^{2}
$$

By identification

$$
\omega=\cos \left(\frac{\theta}{2}\right) \quad v_{i}=\sin \left(\frac{\theta}{2}\right) n_{i}
$$

with the following spherical coordinate for $\underline{n}$

$$
(\phi, \psi) \in] 0, \pi] \times[0,2 \pi], \quad \underline{\mathrm{n}}=\left(\begin{array}{c}
n_{x}=\sin (\phi) \cos (\psi) \\
n_{y}=\sin (\phi) \sin (\psi) \\
n_{z}=\cos (\phi)
\end{array}\right)
$$

In fact we have the following hypersherical coordinates

$$
\left(\begin{array}{c}
x_{t}=R \cos \left(\frac{\theta}{2}\right) \\
x_{z}=R \sin \left(\frac{\theta}{2}\right) \cos (\phi) \\
x_{x}=R \sin \left(\frac{\theta}{2}\right) \sin (\phi) \cos (\psi) \\
x_{y}=R \sin \left(\frac{\theta}{2}\right) \sin (\phi) \sin (\psi)
\end{array}\right)
$$

with $R$ the hyper radius. We can define the radius of the projected sphere by

$$
r=R \sin \left(\frac{\theta}{2}\right)
$$

and the radius of the projected circle

$$
\rho=r \sin (\phi)=R \sin \left(\frac{\theta}{2}\right) \sin (\phi)
$$

in such a way that

$$
\left(\begin{array}{c}
x_{t}=R \cos \left(\frac{\theta}{2}\right) \\
x_{z}=r \cos (\phi) \\
x_{x}=\rho \cos (\psi) \\
x_{y}=\rho \sin (\psi)
\end{array}\right)
$$

For points on the unit hypersphere:

$$
\left\{\begin{array}{l}
R=1 \\
r=\sin \left(\frac{\theta}{2}\right) \\
\rho=r \sin (\phi)=\sin \left(\frac{\theta}{2}\right) \sin (\phi)
\end{array}\right.
$$


Let's take back to the rotation formula

$$
\underline{\mathrm{q}}^{\star}=h \underline{\mathrm{q}} h^{-1}=(\omega+\underline{\mathrm{v}}) \underline{\mathrm{q}}(\omega-\underline{\mathrm{v}})=\omega^{2} \underline{\mathrm{q}}+\omega(\underline{\mathrm{v}} \underline{\mathrm{q}}-\underline{\mathrm{q}} \underline{\mathrm{v}})-\underline{\mathrm{v}} \underline{\mathrm{q}} \underline{\mathrm{v}}
$$

The multiplication of two pure quaternions gives:

$$
\underline{\mathrm{v}} \underline{\mathrm{q}}=-\underline{\mathrm{v}} \cdot \underline{\mathrm{q}}+\underline{\mathrm{v}} \wedge \underline{\mathrm{q}}
$$

Hence

$$
\underline{\mathrm{q}}^{\star}=h \underline{\mathrm{q}} h^{-1}=\left(\omega^{2}+\underline{\mathrm{v}} \cdot \underline{\mathrm{v}}\right) \underline{\mathrm{q}}+2 \omega(\underline{\mathrm{v}} \wedge \underline{\mathrm{q}})+2 \underline{\mathrm{v}} \wedge(\underline{\mathrm{v}} \wedge \underline{\mathrm{q}})=\underline{\mathrm{q}}+2 \omega(\underline{\mathrm{v}} \wedge \underline{\mathrm{q}})+2 \underline{\mathrm{v}} \wedge(\underline{\mathrm{v}} \wedge \underline{\mathrm{q}})
$$

In the $\mathbb{R}^{3}$ canonical basis

$$
Q_{i j}=\underline{\mathrm{q}}_{j}^{\star} \cdot \underline{\mathrm{e}}_{i}=\delta_{i j}+2 \omega\left(\underline{\mathrm{v}} \wedge \underline{\mathrm{e}}_{j}\right) \cdot \underline{\mathrm{e}}_{i}+2 \underline{\mathrm{v}} \wedge\left(\underline{\mathrm{v}} \wedge \underline{\mathrm{e}}_{j}\right) \cdot \underline{\mathrm{e}}_{i}
$$

Using vectorial identities it finally can be established that:

$$
\underset{\sim}{\mathrm{Q}}=\left(\omega^{2}-\|\underline{\mathrm{v}}\|^{2}\right) \underset{\sim}{\mathrm{I}}-2 \underset{\sim}{\epsilon} \cdot \underline{\mathrm{v}}+2 \underline{\mathrm{v}} \otimes \underline{\mathrm{v}}
$$

In matrix form

$$
\underset{\sim}{\mathrm{Q}}=\left(\begin{array}{ccc}
\omega^{2}+v_{x}^{2}-v_{y}^{2}-v_{z}^{2} & 2\left(v_{x} v_{y}-\omega v_{z}\right) & 2\left(\omega v_{y}+v_{x} v_{z}\right) \\
2\left(v_{x} v_{y}+\omega v_{z}\right) & \omega^{2}-v_{x}^{2}+v_{y}^{2}-v_{z}^{2} & 2\left(v_{y} v_{z}-\omega v_{x}\right) \\
2\left(v_{x} v_{z}-\omega v_{y}\right) & 2\left(\omega v_{x}+v_{y} v_{z}\right) & \left(\omega^{2}-v_{x}^{2}-v_{y}^{2}+v_{z}^{2}\right)
\end{array}\right)
$$

If we introduce the polar parametrization of quaternion in (30) we obtain:

$$
\underset{\sim}{\mathrm{Q}}=\cos (\theta) \underset{\sim}{\mathrm{I}}-\sin (\theta) \underset{\underline{\epsilon}}{\sim} \underline{\mathrm{n}}+(1-\cos (\theta)) \underline{\mathrm{n}} \otimes \underline{\mathrm{n}}
$$

which is the well-known Rodrigues formula for rotations.

Using the parametrization (29), equation (22) reads, with $n_{x}^{2}+n_{y}^{2}=\sin ^{2}(\phi)$ and $\rho^{2}=\alpha$ :

$$
\rho^{2}=\sin ^{2}\left(\frac{\theta}{2}\right) \sin ^{2}(\phi)
$$

Moreover, as $\mathrm{n} \in \mathcal{S}^{2}$, we get

$$
n_{z}^{2}=\cos ^{2}(\phi)
$$

Consider first the case $\rho \in] 0,1[$ and suppose that $\phi \neq 0$. In this case the system (32)-(33) can be rewritten as

$$
\left\{\begin{array}{l}
\theta= \pm 2 \arcsin \left(\frac{\rho}{\sin (\phi)}\right) \\
n_{z}= \pm \cos (\phi)
\end{array}\right.
$$

and the set of rotations associated to the parameter $\rho^{2}$ is

$$
\left.\left.\Phi^{-1}\left(\rho^{2}\right)=\left\{\underline{\mathrm{n}}^{ \pm}=\left(\begin{array}{c}
\sin (\phi) \cos (\psi) \\
\sin (\phi) \sin (\psi) \\
\pm \cos (\phi)
\end{array}\right), \theta= \pm 2 \arcsin \left(\frac{\rho}{\sin (\phi)}\right), \forall(\phi, \psi) \in\right] 0, \pi\right] \times[0,2 \pi]\right\}
$$

Consider now the case $\rho=1$. In such a case, system (32)-(33) implies

$$
\sin ^{2}\left(\frac{\theta}{2}\right)=1 \quad ; \quad \sin (\phi)^{2}=1
$$

Hence

$$
\Phi^{-1}(1)=\left\{\underline{\mathrm{n}}^{ \pm}=\left(\begin{array}{c}
\cos (\psi) \\
\sin (\psi) \\
0
\end{array}\right), \theta=(2 k+1) \pi, \forall k \in \mathrm{Z}, \psi \in[0,2 \pi]\right\}
$$


Consider now the case $\rho=0$. The system (32)-(33) reads

$$
\sin ^{2}\left(\frac{\theta}{2}\right) \sin (\phi)^{2}=0
$$

which implies that either $\sin (\phi)^{2}=0$ or $\sin ^{2}\left(\frac{\theta}{2}\right)=0$. The case $\sin (\phi)^{2}=0$ corresponds to a physical rotation:

$$
\Phi^{-1}(0)=\left\{\underline{\mathrm{n}}^{ \pm}=\left(\begin{array}{c}
0 \\
0 \\
\pm 1
\end{array}\right), \theta \in[0,2 \pi]\right\}
$$

while the case $\sin ^{2}\left(\frac{\theta}{2}\right)=0$ corresponds to a "null" rotation around any direction:

$$
\Phi^{-1}(0)=\left\{\underline{\mathrm{n}}^{ \pm} \in S^{2}, \theta=2 k \pi, \forall k \in \mathrm{Z}\right\}
$$

\section{Proofs of propositions 7.4 and 7.5}

First, recall that:

- the tensors considered are real valued, so that the Boehler invariants $\in \mathbb{R}$;

$-I_{2} \geq 0$ and $J_{2} \geq 0$ by definition;

- the elementary symmetric functions can be expressed in terms of Boehler invariants, see (16).

Proof. Proposition 7.4

The condition for an elastic material to have at least 2 equal eigenvalues reads in terms of elementary symmetric functions:

$$
-\sigma_{1}^{2} \sigma_{2}^{2}+4 \sigma_{2}^{3}+4 \sigma_{1}^{3} \sigma_{3}-18 \sigma_{1} \sigma_{2} \sigma_{3}+27 \sigma_{3}^{2}=0
$$

Using (16), the subset of at-least-orthotropic materials with two identical eigenvalues is defined by the algebraic system:

$$
\left\{\begin{array}{l}
2 I_{3}^{2}-I_{2}^{2} J_{2}=0 \\
-\left(I_{1}+J_{1}\right)^{2}\left(I_{2}-2 J_{1}\left(4 I_{1}+J_{1}\right)+J_{2}\right)^{2}-\frac{1}{2}\left(I_{2}-2 J_{1}\left(4 I_{1}+J_{1}\right)+J_{2}\right)^{3}+\ldots \\
+16\left(I_{1}+J_{1}\right)^{3}\left(I_{3}-I_{2} J_{1}+4 I_{1} J_{1}^{2}-2 I_{1} J_{2}\right)-\ldots \\
-9\left(I_{1}+J_{1}\right)\left(-I_{2}+2 J_{1}\left(4 I_{1}+J_{1}\right)-J_{2}\right)\left(I_{3}-I_{2} J_{1}+4 I_{1} J_{1}^{2}-2 I_{1} J 2\right)+\ldots \\
+\frac{27}{4}\left(I_{3}-I_{2} J_{1}+4 I_{1} J_{1}^{2}-2 I_{1} J_{2}\right)^{2}=0
\end{array}\right.
$$

1. For $I_{2}=0,(24)$ and (36) reduce to the same polynomial conditions:

$$
\left\{\begin{array}{l}
I_{3}=0 \\
J_{2}\left(-8 I_{1}^{2}+8 I_{1} J_{1}-2 J_{1}^{2}+J_{2}\right)^{2}=0
\end{array}\right.
$$

2. The case $I_{2} \neq 0$ and $J_{2}=0$ cannot occur as in this case the material is $R_{0}$-orthotropic and have three distinct eigenvalues:

$$
\left\{\begin{array}{l}
\lambda_{1}=J_{1} \\
\lambda_{2}=\frac{1}{2}\left(2 I_{1}+J_{1}-\sqrt{2 I_{2}+\left(2 I_{1}-J_{1}\right)^{2}}\right) \\
\lambda_{3}=\frac{1}{2}\left(2 I_{1}+J_{1}+\sqrt{2 I_{2}+\left(2 I_{1}-J_{1}\right)^{2}}\right)
\end{array}\right.
$$

3. With $I_{2} \neq 0$ and $J_{2} \neq 0, I_{3}= \pm \frac{1}{\sqrt{2}} I_{2} \sqrt{J_{2}}$ (recall that by definition $J_{2} \geq 0$ ). 
(a) Let consider the case $I_{3}=+\frac{1}{\sqrt{2}} I_{2} \sqrt{J_{2}}$. Solutions $J_{1}^{a, b, c}$ of (36) are

$$
J_{1}^{a}=2 I_{1}-\frac{I_{2}-2 J_{2}}{2 \sqrt{2} \sqrt{J_{2}}} \quad J_{1}^{b, c}=2 I_{1}-\frac{\sqrt{J_{2}}}{\sqrt{2}} \pm 2 i \frac{\sqrt{I_{2}}}{\sqrt{2}}
$$

so that $J_{1}^{a}$ is the only real valued solution. On the other hand, the Groebner basis of (24) reads in this case:

$$
-I 2 \frac{\sqrt{J_{2}}}{\sqrt{2}}+4 I_{1} J_{2}-2 J_{1} J_{2}+\sqrt{2} J_{2}^{\frac{3}{2}}=0
$$

for which the unique solution with respect to $J_{1}$ is $J_{1}^{a}$ again.

(b) The case $I_{3}=-\frac{1}{\sqrt{2}} I_{2} \sqrt{J_{2}}$ is similar to the previous case up to some sign changes.

We have thus proved that the real valued solution of (36) are identical to the (real valued) solution of (24). This ends the proof.

Proof. Proposition 7.5

The necessary and sufficient conditions for an elastic material to have at least 2 eigenvalues equal to zero reads in terms of elementary symmetric functions:

$$
\sigma_{2}=0 \quad \text { and } \quad \sigma_{3}=0
$$

Using (16), the subset of at-least-orthotropic material with two zero eigenvalues is defined by the algebraic system:

$$
\left\{\begin{array}{l}
2 I_{3}-I_{2}^{2} J_{2}=0 \\
4 I_{1} J_{1}+J_{1}^{2}-\frac{1}{2}\left(I_{2}+J_{2}\right)=0 \\
I_{3}-I_{2} J_{1}+4 I_{1} J_{1}^{2}-2 I_{1} J_{2}=0
\end{array}\right.
$$

The Groebner basis of the previous algebraic system reads (with lexicographic order $\left(I_{3}, J_{2}, I_{2}, J_{1}, I_{1}\right)$ ):

$$
\left\{\begin{array}{l}
\left(8 I_{1}^{2}+I_{2}\right)\left(I_{2}-8 I_{1} J_{1}\right)^{2}=0 \\
I_{2}-8 I_{1} J_{1}-2 J_{1}^{2}+J_{2}=0 \\
2 I_{1} I_{2}+I_{3}-16 I_{1}^{2} J_{1}-I_{2} J_{1}=0
\end{array}\right.
$$

The case $I_{1}=0$ implies that $I_{2}=0$, so that $J_{2}=2 J_{1}^{2}$ and $I_{3}=0$.

The case $I_{1} \neq 0$ implies that $8 I_{1}^{2}+I_{2} \neq 0$ because $I_{2} \geq 0$, so that $I_{2}=8 I_{1} J_{1}$, and finally $J_{2}=2 J_{1}^{2}$ and $I_{3}=8 I_{1} J_{1}^{2}$. We have thus prooved that (39) implies (26).

It is trivial to check that (26) implies (39). This ends the proof.

\section{On reduced anisotropic invariants}

Let consider the harmonic basis $\mathcal{H}=\left\{\underline{\hat{f}}_{1}, \underline{\hat{f}}_{2}, \hat{\underline{f}}_{3}\right\}$ introduced subsection 3.1, and define the following second order tensors:

$$
\underset{\sim}{\mathrm{d}}:=\underline{\mathrm{f}}_{1} \otimes \underline{\hat{\mathrm{f}}}_{1}+\underline{\mathrm{f}}_{2} \otimes \underline{\hat{\mathrm{f}}}_{2} \quad ; \quad \underset{\sim}{\mathrm{s}}:=\underline{\hat{\mathrm{f}}}_{3} \otimes \underline{\mathrm{f}}_{3}
$$

We can specify the following group of transformations

$$
\text { Dil }:=\{\underset{\sim}{\mathrm{H}} \in \mathrm{GL}(3), \underset{\sim}{\mid \mathrm{H}}=\alpha \underset{\sim}{\mathrm{d}}+\beta \underset{\sim}{\mathrm{s}}\}
$$

which elements have the following matrix form with respect to $\mathcal{H}$

$$
\underset{\sim}{\mathrm{H}}(\alpha, \beta)=\left(\begin{array}{lll}
\alpha & 0 & 0 \\
0 & \alpha & 0 \\
0 & 0 & \beta
\end{array}\right)_{\mathcal{H}}
$$


Consider the following relation amongst elasticity tensors

$$
\underset{\approx}{\mathrm{T}_{1}} \sim \underset{\approx}{\mathrm{T}_{2}} \Leftrightarrow \underset{\sim}{\exists \mathrm{H}} \in \operatorname{Dil} \mid \underset{\sim}{\mathrm{T}_{2}}=\underset{\sim}{\mathrm{H}} \star \underset{\approx}{\mathrm{T}}
$$

This relation can be shown to be : reflexive, symmetric and transitive. In other words, this is an equivalence relation. The equivalence class of an element $\mathrm{T} \in \mathbb{E}$ la is defined as

$$
\underset{\approx}{\left[\mathrm{T}_{1}\right]}=\left\{\underset{\approx}{\mathrm{T}} \in \mathbb{E} \text { la }, \underset{\sim}{\exists \mathrm{H}} \in \operatorname{Dil} \mid \underset{\approx}{\mathrm{T}}=\underset{\sim}{\mathrm{H}} \star \underset{\approx}{\mathrm{T}} \mathrm{T}_{1}\right\}
$$

The equivalence class can also be defined as the (non-compact) orbit of $\underset{\approx}{\mathrm{T}_{1}}$ with respect to Dil. As well-known the space $\mathbb{E}$ la can be partitioned into disjoint sets of equivalence classes. It can be observed that the adimensional anisotropic invariants:

$$
i_{2}=\frac{I_{2}}{8 I_{1} J_{1}} \quad j_{2}=\frac{J_{2}}{2 J_{1}^{2}} \quad i_{3}=\frac{I_{3}}{8 I_{1} J_{1}^{2}}
$$

introduced in section 5 are constant for the equivalence classes introduced above. For example, let consider a tensor $\mathrm{T}_{1}$ having $\left(i_{2}, j_{2}, i_{3}\right)$ as adimensional invariants and that the associated elastic material is defined by $\left(I_{1}, J_{2}, I_{2}, J_{2}, I_{3}\right)$. A tensor $\underset{\approx}{\mathrm{T}_{2}}$ in the same equivalence class is associated to the elastic material $\left(\beta^{2} I_{1}, \alpha^{2} J_{1}, \alpha^{2} \beta^{2} I_{2}, \alpha^{4} \underset{J_{2}}{\approx}, \alpha^{4} \beta^{2} I_{3}\right)$, a direct computation shows that the associated adimensional invariants will remain unchanged. The converse is not true, since different tensors (and not elastic materials) can have the same set of reduced adimensional invariants without being related by the equivalence relation. For example, consider $\mathrm{T}_{1}$ and $\mathrm{T}_{2}$ two tensors which are conjugated module $\mathrm{SO}(2)$. Those tensors possess the same set of adimensional invariants but are not related by any element of Dil.

\section{References}

1. Y. Arramon, M. Mehrabadi, D. Martin, and S. C. Cowin. A multidimensional anisotropic strength criterion based on Kelvin modes. International Journal of Solids and Structures, $37: 2915-2935,2000$.

2. N. Auffray. Gomtrie des espaces de tenseurs, application l'lasticit anisotrope classique et gnralise. Habilitation diriger des recherches, Universit Paris-Est, 2017.

3. N. Auffray, B. Kolev, and M. Olive. Handbook of bidimensional tensors: Part I: Decomposition and symmetry classes. Mathematics and Mechanics of Solids, page 1081286516649017 , 2016.

4. N. Auffray, B. Kolev, and M. Petitot. On anisotropic polynomial relations for the elasticity tensor. Journal of Elasticity, 115:77-103, 2014.

5. N. Auffray and P. Ropars. Invariant-based reconstruction of bidimensionnal elasticity tensors. International Journal of Solids and Structures, 87:183-193, 2015.

6. M. Biegler and M. Mehrabadi. An energy-based constitutive model for anisotropic solids subject to damage. Mech. Mater., 19:151-164, 1995.

7. A. Blinowski, J. Ostrowska-Maciejewska, and J. Rychlewski. Two-dimensional Hooke's tensors Isotropic decomposition, effective symmetry criteria. Archives of Mechanics, 48:325-345, 1996.

8. J. Boehler. Lois de comportement anisotrope des milieux continus. J. Mcanique, 17(2):153$190,1978$.

9. J.-P. Boehler, A. Kirillov, and E. Onat. On the polynomial invariants of the elasticity tensor. Journal of Elasticity, 34(2):97-110, 1994.

10. B. Desmorat and G. Duvaut. Compliance optimization with nonlinear elastic materials: Application to constitutive laws dissymmetric in tension-compression. European Journal of Mechanics A/Solids, 22:179-192, 2003.

11. B. Desmorat and P. Vannucci. An alternative to the Kelvin decomposition for plane anisotropic elasticity. Mathematical Methods in the Applied Sciences, 38:164-175, 2015.

12. R. Desmorat. Dissymtrie de comportement lastique anisotrope coupl ou non l'endommagement. Comptes Rendus de l'Acadmie des Sciences - Series IIB - Mechanics, 328:445-450, 2000. 
13. R. Desmorat. Kelvin decomposition and multiple effective stresses concept in anisotropic materials. Comptes-Rendus Mcanique, 337:733-738, 2009.

14. R. Desmorat and R. Marull. Non-quadratic Kelvin modes based plasticity criteria for anisotropic materials. International Journal of Plasticity, 27:328-351, 2011.

15. M. Franois. Identification des symtries matrielles de matriaux anisotropes. $\mathrm{PhD}$ Thesis, Universit Paris 6, Pierre et Marie Curie, 1995.

16. M. Franois. A damage model based on Kelvin eigentensors and Curie principle. Mechanics of Materials, 44(0):23 - 34, 2012.

17. M. Golubitsky, I. Stewart, and D. Schaeffer. Singularities and groups in bifurcation theory. Vol. II, volume 69 of Applied Mathematical Sciences. Springer-Verlag, New York, 1988.

18. Q.-C. He and Q.-S. Zheng. On the symmetries of $2 \mathrm{~d}$ elastic and hyperelastic tensors. Journal of elasticity, 43:203-225, 1996.

19. A. Mattiello, R. Desmorat, and J. Cormier. Rate dependent ductility and damage threshold: Application to Nickel-based single crystal CMSX-4. International Journal of Plasticity, 2018.

20. M. M. Mehrabadi and S. Cowin. Eigentensors Of Linear Anisotropic Elastic Materials. The Quarterly Journal of Mechanics and Applied Mathematics, 43:15-41, 1990.

21. M. Olive and N. Auffray. Isotropic invariants of completely symmetric third-order tensors. Journal of Mathematical Physic, 55:092901, 2014.

22. M. Olive, B. Kolev, and N. Auffray. A minimal integrity basis for the elasticity tensor. Archive for Rational Mechanics and Analysis, 226(1):1-31, 2017.

23. K. Petersen and M. Pedersen. The matrix cookbook. Technical University of Denmark, $7(15): 510,2008$

24. R. Rivlin. Further Remarks on the Stress-Deformation Relation for Isotropic Materials. Journal of Rational Mechanics and Analysis, 4:681-701, 1955.

25. J. Rychlewsky. On Hooke's law. Prikl Mat Mekh, 48:420-435, 1984.

26. G. Smith. On isotropic functions of symmetric tensors, skew-symmetric tensors and vectors. International Journal of Engineering Science, 9:899-916, 1971.

27. W. Thomson (Lord Kelvin). Elements of a mathematical theory of elasticity. Phil. Trans. R. Soc., 166:481, 1856

28. P. Vannucci. A special planar orthotropic material. Journal of Elasticity, 67:81-96, 2002.

29. P. Vannucci. Plane anisotropy by the polar method. Meccanica, 40:437-454, 2005.

30. G. Verchery. Les invariants des tenseurs d'ordre 4 du type de l'lasticit. In CNRS, editor, Euromech 115, volume 31, pages 93-104, 1982.

31. M. Vianello. An integrity basis for plane elasticity tensors. Archives of Mechanics, 49:197208, 1997.

32. H. Weyl. The classical groups. Princeton Landmarks in Mathematics. Princeton University Press, Princeton, NJ, 1997.

33. Q.-S. Zheng. Theory of representations for tensor functions - A unified invariant approach to constitutive equations. Applied Mechanics Reviews, 47:545-587, 1994.

34. Q.-S. Zheng and J.-P. Boehler. The description, classification, and reality of material and physical symmetries. Acta Mechanica, 102(1-4):73-89, 1994. 